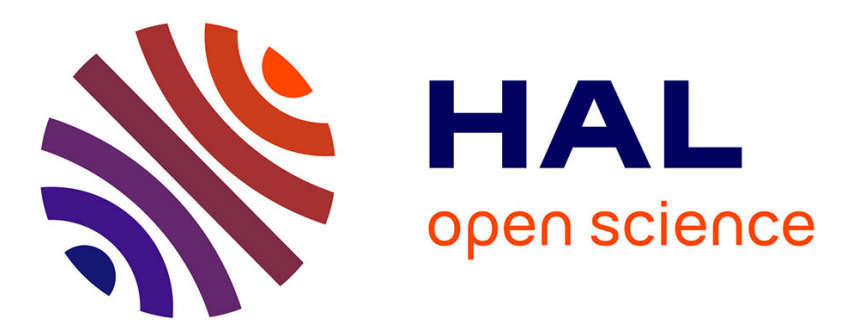

\title{
A discrete duality finite volume discretization of the vorticity-velocity-pressure formulation of the 2D Stokes problem on almost arbitrary two-dimensional grids
}

\author{
Sarah Delcourte, Pascal Omnes
}

\section{> To cite this version:}

Sarah Delcourte, Pascal Omnes. A discrete duality finite volume discretization of the vorticity-velocitypressure formulation of the 2D Stokes problem on almost arbitrary two-dimensional grids. Numerical Methods for Partial Differential Equations, 2015, pp.1-30. 10.1002/num.21890 . cea-00772972v2

\section{HAL Id: cea-00772972}

https://hal-cea.archives-ouvertes.fr/cea-00772972v2

Submitted on 25 Jul 2014

HAL is a multi-disciplinary open access archive for the deposit and dissemination of scientific research documents, whether they are published or not. The documents may come from teaching and research institutions in France or abroad, or from public or private research centers.
L'archive ouverte pluridisciplinaire HAL, est destinée au dépôt et à la diffusion de documents scientifiques de niveau recherche, publiés ou non, émanant des établissements d'enseignement et de recherche français ou étrangers, des laboratoires publics ou privés. 


\title{
A discrete duality finite volume discretization of the vorticity-velocity-pressure Stokes problem on almost arbitrary two-dimensional grids
}

\author{
Sarah Delcourte ${ }^{1}$ and Pascal Omnes ${ }^{23}$
}

\begin{abstract}
We present an application of the discrete duality finite volume method to the numerical approximation of the vorticity-velocity-pressure formulation of the 2D Stokes equations, associated to various non-standard boundary conditions. The finite volume method is based on the use of discrete differential operators obeying some discrete duality principles. The scheme may be seen as an extension of the classical MAC scheme to almost arbitrary meshes, thanks to an appropriate choice of degrees of freedom. The efficiency of the scheme is illustrated by numerical examples over unstructured triangular and locally refined nonconforming meshes, which confirm the theoretical convergence analysis led in the article.
\end{abstract}

Keywords: Stokes equations; vorticity-velocity-pressure formulation; boundary conditions; discrete duality; finite volumes; arbitrary meshes; a priori estimates

\section{Introduction}

Let $\Omega$ be a bounded, open, connected but not necessarily simply connected polygon of $\mathbb{R}^{2}$, whose boundary is denoted by $\Gamma$; we consider the numerical approximation by means of finite volumes of the solution of the Stokes equations: with given functions $\mathbf{f}, g, \sigma, p_{d}$ and $\omega_{d}$ and a given real number $m_{\omega}$, find $(\mathbf{u}, p)$ satisfying

$$
\begin{aligned}
-\boldsymbol{\Delta u}+\boldsymbol{u} p & =\mathbf{f} \text { in } \Omega, \\
\nabla \cdot \mathbf{u} & =g \text { in } \Omega,
\end{aligned}
$$

supplemented with one of the following non-standard sets of conditions

$$
\begin{gathered}
\mathbf{u} \cdot \mathbf{n}=\sigma \text { over } \Gamma, \quad \nabla \times \mathbf{u}=\omega_{d} \text { over } \Gamma \text { and } \int_{\Omega} p(\mathbf{x}) d \mathbf{x}=0 \\
\mathbf{u} \cdot \mathbf{n}=\sigma \text { over } \Gamma \quad, \quad p=p_{d} \text { over } \Gamma \text { and } \int_{\Omega} \nabla \times \mathbf{u}(\mathbf{x}) d \mathbf{x}=m_{\omega}, \\
\mathbf{u} \cdot \boldsymbol{\tau}=\sigma \text { over } \Gamma, \quad \nabla \times \mathbf{u}=\omega_{d} \text { over } \Gamma \quad \text { and } \quad \int_{\Omega} p(\mathbf{x}) d \mathbf{x}=0, \\
\mathbf{u} \cdot \boldsymbol{\tau}=\sigma \text { over } \Gamma \quad, \quad p=p_{d} \text { over } \Gamma \text { and } \int_{\Omega} \nabla \times \mathbf{u}(\mathbf{x}) d \mathbf{x}=m_{\omega} .
\end{gathered}
$$

\footnotetext{
${ }^{1}$ Université de Lyon, CNRS UMR 5208, Université Lyon 1, Institut Camille Jordan, 43 boulevard du 11 novembre 1918, F-69622 Villeurbanne cedex, France. Partially supported by the European Research Council ERC Starting Grant 2009, project 239983-NuSiKiMo. delcourte@math.univ-lyon1.fr

${ }^{2}$ CEA-Saclay, DEN, DM2S, STMF, LMSF, F-91191 Gif-sur-Yvette, France. E-Mail: pascal.omnes@cea.fr

${ }^{3}$ Université Paris 13, Sorbonne Paris Cité, LAGA, CNRS UMR 7539, Institut Galilée, 99, Avenue J.-B. Clément F-93430 Villetaneuse Cedex, France.
} 
These conditions are written here in the case of simply connected domains, but they will be extended in the core of the article to non-simply connected domains. There are compatibility conditions between the data $(g, \sigma)$ in $(1.3)$ and $(1.4)$, the data $\left(\omega_{d}, \sigma\right)$ in (1.5) and the data $\left(m_{\omega}, \sigma\right)$ in (1.6). They will be discussed in subsection 4.1.

As recognized for example by Dubois et. al. [1], these non-standard conditions can be treated in a very general and natural way thanks to the vorticity-velocity-pressure formulation of the Stokes problem (for earlier works based on different approaches, we refer to [2] and [3]). Since $-\boldsymbol{\Delta} \mathbf{u}=\boldsymbol{\nabla} \times \nabla \times \mathbf{u}-\boldsymbol{\nabla} \nabla \cdot \mathbf{u}$, using (1.2), and introducing the vorticity $\omega$, Eq. (1.1) may be split as

$$
\begin{aligned}
\boldsymbol{\nabla} \times \omega+\boldsymbol{\nabla} p & =\mathbf{f}+\boldsymbol{\nabla} g \text { in } \Omega, \\
\nabla \times \mathbf{u} & =\omega \text { in } \Omega .
\end{aligned}
$$

The mathematical analysis of system (1.7)-(1.8)-(1.2) with various boundary conditions was led in several references, among which $[4,5,6,7,1]$. Finite element methods for the vorticityvelocity-pressure formulation were analyzed in $[4,5,8,9]$. Spectral methods were considered in $[6,10]$ and $[11]$, where a least-square formulation is used.

In the present work, we shall be interested in a finite volume generalization of the Marker and Cell (MAC) scheme on very general meshes. The MAC scheme was developed initially in [12] on staggered rectangular grids and extended to the so-called covolume scheme using Delaunay-Voronoi mesh pairs, as reviewed in [13]. We note that the orthogonality property of these mesh pairs might be in certain cases a drawback, in particular in the context of adaptive mesh refinement. The standard MAC scheme discretizes (1.1)-(1.2), while the covolume scheme discretizes (1.7)-(1.8)-(1.2). Given a (primal) mesh, the MAC and covolume schemes use as velocity unknowns the normal components of the velocity field with respect to the edges of the control volumes, while the pressure unknowns are located at their circumcenters. In addition, the covolume scheme considers vorticity unknowns at the vertices of the primal mesh.

The generalization of the MAC scheme we propose is a new application of the "discrete duality finite volume" (DDFV) method [14]. Originally developed for linear diffusion equations [15, $16,17]$, the DDFV method was extended to nonlinear diffusion $[18,19,20]$, convection-diffusion [21], electro-cardiology [22, 23], drift-diffusion and energy-transport models [24], electro- and magnetostatics [25], electromagnetism [26], and Stokes flows [27, 28, 29, 30]. The advantage of this covolume-like method is that it allows the use of almost arbitrary meshes, including very distorted, degenerating, or highly non-conforming meshes (see the numerical tests in [15]). The name of the method comes from the definition of discrete gradient and divergence operators verifying a discrete Green formula.

In order to get rid of the orthogonality constraints that restrict the use of MAC and covolume schemes to certain families of meshes, the price to pay in the DDFV framework is to discretize both velocity components on the edges of the control volumes, while pressure and vorticity unknowns are associated with the centroids of the primal cells and to their vertices. Then, we integrate both components of (1.7) on cells associated with the edges (the so-called "diamond mesh") and (1.8) and (1.2) on both primal and dual cells associated to the vertices. This process uses discrete versions of the divergence, gradient and curl operators which appear in (1.7), (1.8) and (1.2). These discrete operators are known to satisfy properties analogous to those verified by the continuous operators [25]. With these properties, we show that the solution of the DDFV 
discretization of the Stokes equations with any of the conditions (1.3) to (1.6) can be reduced to the solution of four discrete Laplace equations involving the pressure, the vorticity and the potentials stemming from the discrete Hodge decomposition of the velocity.

Other approaches with finite volumes were developed in [31, 32, 33, 34]. In [31, 32], velocity unknowns are located at the cell centers, and pressure unknowns at the vertices. But an orthogonality condition is also needed: the segments connecting the cell centers must be orthogonal to the edges of the mesh, which restricts this scheme to particular families of meshes. The same kind of orthogonality condition is needed in [33, 34], where velocity and pressure unknowns are all located at the cell centers. This choice requires some stabilization obtained by penalizing the discrete incompressibility equation. Such a penalization is also needed in the previous approaches that used the DDFV ideas $[28,29,30]$ because in these works, velocity unknowns are located at the cell centers and vertices, while pressure is defined on the edges (or faces in $3 \mathrm{D}$ ) of the cells, and thus the pressure gradient is proved to be injective only on simplices, and there might be spurious pressure modes on more general meshes if no penalization is added. In the approach developed in the present article, such a penalization is not needed: The pressure gradient is computed from two pressure differences, one related to pressure unknowns located at the vertices of an edge, and another related to pressure unknowns located at two neighboring cell centers. This leads to the fact that on any mesh, a vanishing gradient implies that pressure is a constant on the primal and on the dual meshes. Moreover, in our work, the important role played in the Helmholtz-Hodge decomposition of vector fields by internal boundaries in the case of non-simply connected domains is very simply taken into account through the Euler formula that links the number of edges, cells, vertices and internal boundaries (see [25] for more details on this). This wouldn't have been the case if we had chosen the same unknown locations as in $[28,29,30]$.

Another important result we obtain here is that there exists a way to discretize the data $\mathbf{f}$ such that if $g=0$ and if $\mathbf{f}$ is the gradient of the pressure (resp. the curl of the vorticity), then our scheme computes exactly (up to the precision of 1D quadrature formulas) the pressure and a vanishing vorticity (resp. the vorticity and a vanishing pressure) at the cell centers and vertices.

As compared to previous work with DDFV schemes, the novelty here is to realize that the vorticity-velocity-pressure formulation of the Stokes equations associated to an appropriate choice of the degrees of freedom reduces the numerical solution of this problem to four discrete DDFV Laplace equations. Another novelty is that in the course of the numerical analysis of the scheme, we are led to evaluate the difference between some functions and their pointwise values on the one hand, and their mean-value projections on the other hand, both on the primal and dual meshes. These kinds of estimations had never been used previously in the DDFV context. Moreover, we had to combine this with some Poincaré inequalities which have recently been proved [35].

When $g=0$ in Eq. (1.2), another feature of this scheme is that it satisfies the notion of "reinforced incompressibility" introduced in a finite volume element context in [36] and in a finite element context in [37] to overcome non-perfectly divergence free velocity modes that may appear in unsteady Navier-Stokes simulations performed with Crouzeix-Raviart [38] finite elements of lowest order. Indeed, since these elements involve pressure unknowns located at the triangle barycenters only, the incompressibility constraint is satisfied only around these barycenters, but the discrete velocity divergence, when computed around the vertices of the mesh, may not vanish 
or even be small. A cure to this problem, proposed in [37] and [36], is to add pressure unknowns at the vertices of the mesh, which introduces incompressibility constraints around these nodes. Thus, on triangular meshes, the scheme presented here has exactly the same unknowns as (but is however not equivalent to) those involved in [37] and [36], and incompressibility conditions are written on each triangle (primal cell) and around each vertex (dual cell) of the mesh. The advantage of the scheme we present here is that it handles meshes that are much more general than triangular.

The convergence analysis shows that pressure, vorticity, their gradients and the velocity field are all first order accurate on general meshes. Moreover, for families of meshes where diamond cells are almost all parallelograms, pressure and vorticity gradients superconverge with the order 1.5. The numerical tests included in this work validate these theoretical results, and even go beyond: We observe that pressure and vorticity converge on general meshes with the order 2, while velocity converges with the order 2 for families of triangular meshes or for which the diamond cells are almost all parallelograms.

The paper is organized as follows: in Section 2, we introduce the notations associated with the primal, dual and diamond meshes. In Section 3, we define the discrete gradient and vector curl on the diamond cells, and the corresponding adjoint discrete divergence and scalar curl on the primal and dual cells and state their properties. In Section 4, we present the DDFV schemes for the steady Stokes problems with the various conditions (1.3)-(1.6) extended to non-simply connected domains. Section 5 is devoted to the convergence analysis of the DDFV scheme with conditions (1.3). Finally, section 6 presents numerical results over unstructured and non-conforming meshes.

\section{Definitions and notations}

Let $\Omega$ be a bounded connected polygon of $\mathbb{R}^{2}$, with boundary denoted by $\Gamma$. We suppose that the domain has $Q$ holes. Throughout the paper, we shall assume that $Q>0$, but the results also hold for the case $Q=0$. Let $\Gamma_{0}$ denote the exterior boundary of $\Omega$ and let $\Gamma_{q}$, with $q \in[1, Q]$, be the interior polygonal boundaries of $\Omega$, so that $\Gamma=\Gamma_{0} \bigcup_{q \in[1, Q]} \Gamma_{q}$.

\subsection{Construction of the primal mesh}

We consider a first partition of $\Omega$ (named primal mesh) composed of elements $T_{i}$, with $i \in[1, I]$, supposed to be convex polygons. With each element $T_{i}$ of the mesh, we associate a node $G_{i}$ located at the barycentre of $T_{i}$. The area of $T_{i}$ is denoted by $\left|T_{i}\right|$. We shall denote by $J$ the total number of edges of this mesh and by $J^{\Gamma}$ the number of these edges which are located on the boundary $\Gamma$ and we associate with each of these boundary edges its midpoint, also denoted by $G_{i}$ with $i \in\left[I+1, I+J^{\Gamma}\right]$. By a slight abuse of notations, we shall write $i \in \Gamma_{q}$ if and only if $G_{i} \in \Gamma_{q}$. 


\subsection{Construction of the dual mesh}

We denote by $S_{k}$, with $k \in[1, K]$, the nodes of the polygons of the primal mesh. Around each $S_{k}$, we construct a polygon $P_{k}$ by joining the points $G_{i}$ associated to the elements of the primal mesh (and possibly to the boundary edges) of which $S_{k}$ is a node, through the midpoints of the edges. The area of $P_{k}$ is denoted by $\left|P_{k}\right|$. The family of cells $\left(P_{k}\right)_{k \in[1, K]}$ constitutes a second partition of $\Omega$, which we name dual mesh. We shall also write $k \in \Gamma_{q}$ (respectively $k \in \Gamma$ ) if and only if $S_{k} \in \Gamma_{q}$ (resp. $S_{k} \in \Gamma$ ). Figure 1 displays an example of a non-conforming primal mesh and its associated dual mesh.

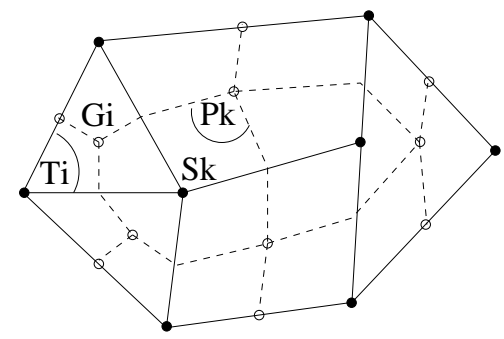

Figure 1: An example of a primal mesh and its associated dual mesh.

\subsection{Construction of the diamond mesh}

With each edge of the primal mesh, denoted by $A_{j}$ (whose length is $\left|A_{j}\right|$ ), with $j \in[1, J]$, we associate a quadrilateral named "diamond cell", denoted by $D_{j}$. When $A_{j}$ is not on the boundary, this cell is obtained by joining the points $S_{k_{1}(j)}$ and $S_{k_{2}(j)}$, which are the two nodes of $A_{j}$, with the points $G_{i_{1}(j)}$ and $G_{i_{2}(j)}$ associated with the elements of the primal mesh which share this edge. When $A_{j}$ is on the boundary $\Gamma$, the cell $D_{j}$ is obtained by joining the two nodes of $A_{j}$ with the point $G_{i_{1}(j)}$ associated with the only element of the primal mesh of which $A_{j}$ is an edge and to the point $G_{i_{2}(j)}$ associated with $A_{j}$ (i.e. by convention $i_{2}(j)$ is element of $\left[I+1, I+J^{\Gamma}\right]$ when $A_{j}$ is located on $\left.\Gamma\right)$. The cells $D_{j}$ constitute the "diamond-mesh", which is a third partition of $\Omega$. The area of the cell $D_{j}$ is denoted by $\left|D_{j}\right|$. Such cells are displayed on Fig. 2. We shall also write $j \in \Gamma_{q}$ (respectively $j \in \Gamma$ ) if and only if $A_{j} \subset \Gamma_{q}$ (resp. $j \subset \Gamma$ ).

\subsection{Definitions of geometrical elements}

The following geometrical elements are displayed on Fig. 3. The unit vector normal to $A_{j}$ is denoted by $\mathbf{n}_{j}$ and is oriented so that its dot product with $\mathbf{G}_{\mathbf{i}_{\mathbf{1}}(\mathbf{j})} \mathbf{G}_{\mathbf{i}_{\mathbf{2}}(\mathbf{j})}$ is positive. We further denote by $A_{j}^{\prime}$ the segment $\left[G_{i_{1}(j)} G_{i_{2}(j)}\right]$ (whose length is $\left|A_{j}^{\prime}\right|$ ) and by $\mathbf{n}_{j}^{\prime}$ the unit vector normal to $A_{j}^{\prime}$ oriented so that $\mathbf{S}_{\mathbf{k}_{\mathbf{1}}(\mathbf{j})} \mathbf{S}_{\mathbf{k}_{\mathbf{2}}(\mathbf{j})} \cdot \mathbf{n}_{j}^{\prime} \geq 0$. We also define the unit vectors $\boldsymbol{\tau}_{j}$ and $\boldsymbol{\tau}_{j}^{\prime}$ such that $\left(\mathbf{n}_{j}, \boldsymbol{\tau}_{j}\right)$ and $\left(\mathbf{n}_{j}^{\prime}, \boldsymbol{\tau}_{j}^{\prime}\right)$ are orthogonal positively oriented bases of $\mathbb{R}^{2}$. We denote by $M_{j}$ the midpoint of $A_{j}$ and by $A_{j 1}^{\prime}$ (respectively $A_{j 2}^{\prime}$ ) the segment $\left[G_{i_{1}(j)} M_{j}\right]$ (resp. $\left[M_{j} G_{i_{2}(j)}\right]$ ) and by $\mathbf{n}_{j 1}^{\prime}$ (resp. $\mathbf{n}_{j 2}^{\prime}$ ) the unit normal vector to $A_{j 1}^{\prime}$ (resp. $A_{j 2}^{\prime}$ ) oriented so that $\left|A_{j}^{\prime}\right| \mathbf{n}_{j}^{\prime}=\left|A_{j 1}^{\prime}\right| \mathbf{n}_{j 1}^{\prime}+\left|A_{j 2}^{\prime}\right| \mathbf{n}_{j 2}^{\prime}$. We define for each $i \in[1, I]$ the set $V(i)$ of integers $j \in[1, J]$ such 

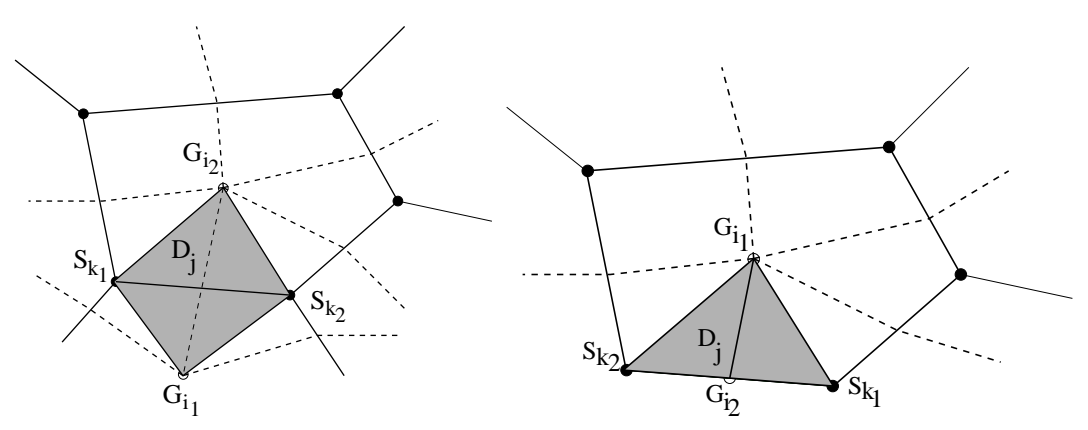

Figure 2: Examples of diamond cells.

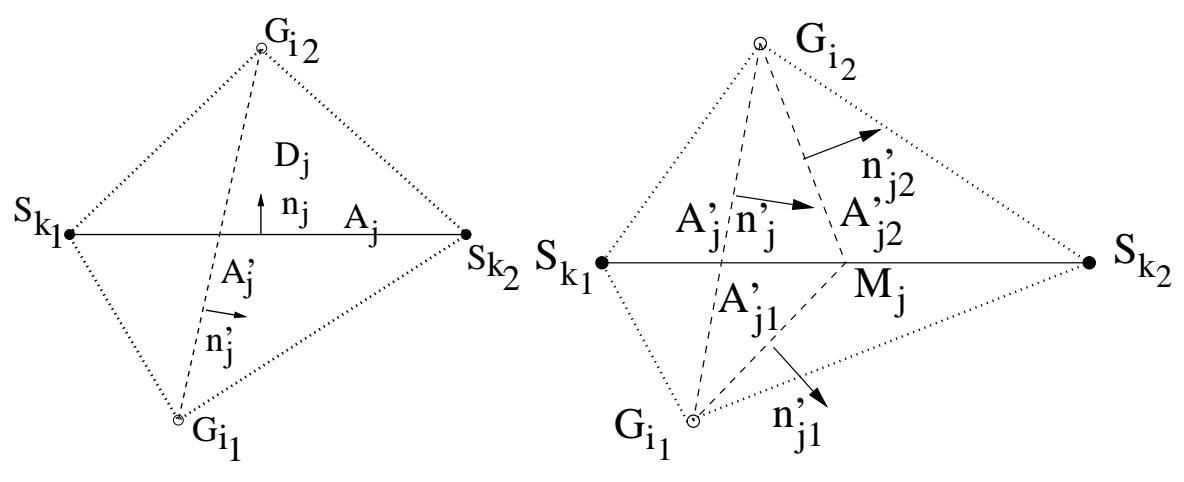

Figure 3: Notations for the diamond cell.

that $A_{j}$ is an edge of $T_{i}$ and for each $k \in[1, K]$ the set $E(k)$ of integers $j \in[1, J]$ such that $S_{k}$ is a node of $A_{j}$.

We define for each $j \in[1, J]$ and each $k$ such that $j \in E(k)$ (resp. each $i$ such that $j \in V(i)$ ) the real-valued number $s_{j k}^{\prime}$ (resp. $s_{j i}$ ) whose value is +1 or -1 whether $\mathbf{n}_{j}^{\prime}$ (resp. $\mathbf{n}_{j}$ ) points outward or inward $P_{k}$ (resp. $\left.T_{i}\right)$. We define $\mathbf{n}_{j k}^{\prime}:=s_{j k}^{\prime} \mathbf{n}_{j}^{\prime}\left(\right.$ resp. $\mathbf{n}_{j i}:=s_{j i} \mathbf{n}_{j}$ ) and remark that $\mathbf{n}_{j k}^{\prime}$ (resp. $\mathbf{n}_{j i}$ ) always points outward $P_{k}\left(\right.$ resp. $\left.T_{i}\right)$. In the same way, we set $\mathbf{n}_{j k 1}^{\prime}:=s_{j k}^{\prime} \mathbf{n}_{j 1}^{\prime}$ and $\mathbf{n}_{j k 2}^{\prime}:=s_{j k}^{\prime} \mathbf{n}_{j 2}^{\prime}$.

\subsection{A hypothesis on the mesh regularity}

In the sequel of this article, we shall obtain error estimates under the following hypothesis (see Figure 4 for the notations).

Hypothesis 2.1. The angles of the subtriangulation $G_{i} M_{j} S_{k}$ of the diamond-cells $D_{j}$ are greater than an angle $\theta^{*}$ which is strictly positive and independent of the mesh:

$$
\exists \theta^{*}, \quad 0<\theta^{*}<\frac{\pi}{2} \quad \text { such that } \quad \min \left\{\alpha_{m}, \beta_{m}, \gamma_{m}\right\} \geq \theta^{*}, \quad m=1,2,3,4 .
$$




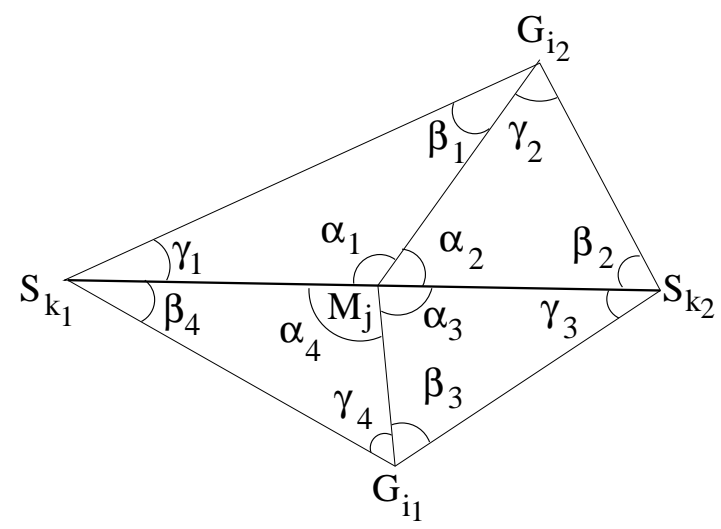

Figure 4: Notations for Hypothesis 2.1.

We can easily verify that Hypothesis 2.1 implies various weaker hypotheses: [15, Hypotheses 5.5 and 5.6], [25, Hypothesis 5.5] and [35, Definition 2.1] under which results obtained in these articles, and used in the present work, are valid.

\section{The discrete operators and their properties}

We may approach the gradient $\boldsymbol{\nabla}=\left(\frac{\partial}{\partial x}, \frac{\partial}{\partial y}\right)^{T}$ and the vector curl $\boldsymbol{\nabla} \times=\left(\frac{\partial}{\partial y},-\frac{\partial}{\partial x}\right)^{T}$ by discrete counterparts on the diamond cells $D_{j}$ (see $\left.[15,25]\right)$.

Definition 3.1. For any $\phi=\left(\phi_{i}^{T}, \phi_{k}^{P}\right) \in \mathbb{R}^{I+J^{\Gamma}} \times \mathbb{R}^{K}$, the discrete gradient $\nabla_{h}^{D}$ and vector curl $\nabla_{h}^{D} \times$ are defined by their values over the diamond cells $D_{j}$ (see Fig. 3):

$$
\begin{array}{r}
\left(\nabla_{h}^{D} \phi\right)_{j}:=\frac{1}{2\left|D_{j}\right|}\left\{\left[\phi_{k_{2}}^{P}-\phi_{k_{1}}^{P}\right]\left|A_{j}^{\prime}\right| \mathbf{n}_{j}^{\prime}+\left[\phi_{i_{2}}^{T}-\phi_{i_{1}}^{T}\right]\left|A_{j}\right| \mathbf{n}_{j}\right\}, \\
\left(\nabla_{h}^{D} \times \phi\right)_{j}:=-\frac{1}{2\left|D_{j}\right|}\left\{\left[\phi_{k_{2}}^{P}-\phi_{k_{1}}^{P}\right]\left|A_{j}^{\prime}\right| \boldsymbol{\tau}_{j}^{\prime}+\left[\phi_{i_{2}}^{T}-\phi_{i_{1}}^{T}\right]\left|A_{j}\right| \boldsymbol{\tau}_{j}\right\} .
\end{array}
$$

Note that formulas (3.1)-(3.2) are exact for any affine function $\phi$ if we set $\phi_{k}^{P}:=\phi\left(S_{k}\right)$ and $\phi_{i}^{T}:=\phi\left(G_{i}\right)$, for any $(i, k)$.

Next, for a discrete vector field $\mathbf{u}$, given by its values $\mathbf{u}_{j}$ on the cells $D_{j}$, we define its discrete divergence and curl on the primal and dual cells.

Definition 3.2. For $\mathbf{u}=\left(\mathbf{u}_{j}\right) \in\left(\mathbb{R}^{2}\right)^{J}$, the discrete divergence $\nabla_{h}^{T, P} \cdot:=\left(\nabla_{h}^{T} \cdot, \nabla_{h}^{P} \cdot\right)$ and discrete curl $\nabla_{h}^{T, P} \times:=\left(\nabla_{h}^{T} \times, \nabla_{h}^{P} \times\right)$ are defined by their values over the primal cells $T_{i}$ and the dual 

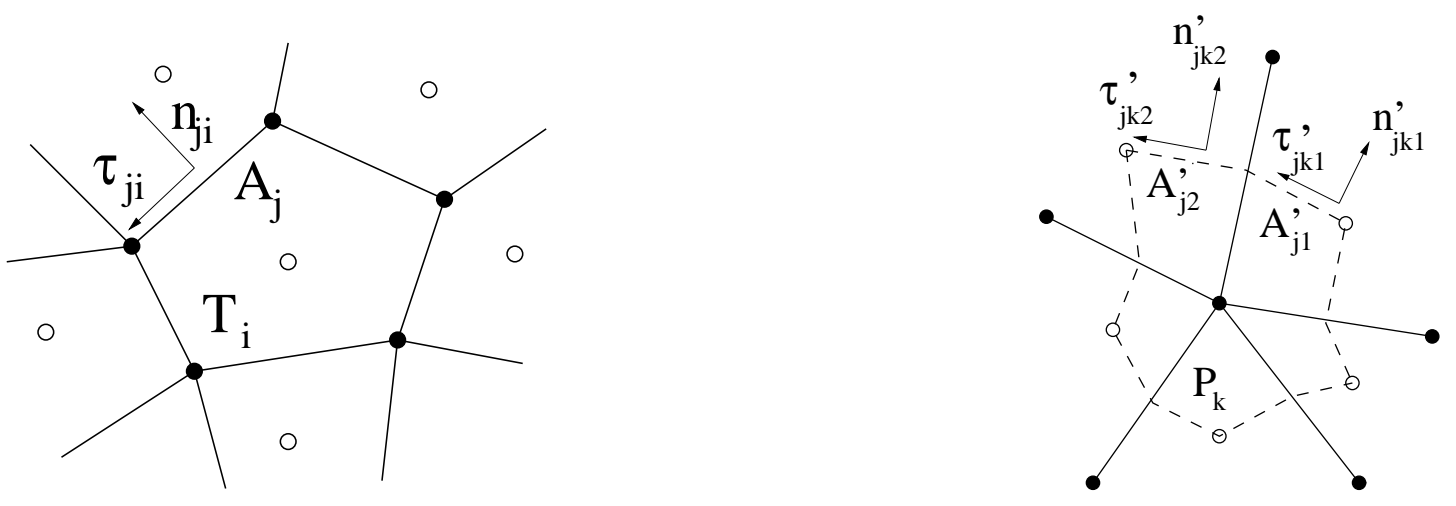

Figure 5: Edges and unit vectors for the discrete divergence and curl

cells $P_{k}$ (see Fig. 5)

$$
\begin{aligned}
\left(\nabla_{h}^{T} \cdot \mathbf{u}\right)_{i} & :=\frac{1}{\left|T_{i}\right|} \sum_{j \in V(i)}\left|A_{j}\right| \mathbf{u}_{j} \cdot \mathbf{n}_{j i}, \\
\left(\nabla_{h}^{P} \cdot \mathbf{u}\right)_{k} & :=\frac{1}{\left|P_{k}\right|}\left(\sum_{j \in E(k)}\left(\left|A_{j 1}^{\prime}\right| \mathbf{n}_{j k 1}^{\prime}+\left|A_{j 2}^{\prime}\right| \mathbf{n}_{j k 2}^{\prime}\right) \cdot \mathbf{u}_{j}+\sum_{j \in E(k) \cap \Gamma} \frac{1}{2}\left|A_{j}\right| \mathbf{u}_{j} \cdot \mathbf{n}_{j}\right) . \\
\left(\nabla_{h}^{T} \times \mathbf{u}\right)_{i}: & =\frac{1}{\left|T_{i}\right|} \sum_{j \in V(i)}\left|A_{j}\right| \mathbf{u}_{j} \cdot \boldsymbol{\tau}_{j i}, \\
\left(\nabla_{h}^{P} \times \mathbf{u}\right)_{k}: & =\frac{1}{\left|P_{k}\right|}\left(\sum_{j \in E(k)}\left(\left|A_{j 1}^{\prime}\right| \boldsymbol{\tau}_{j k 1}^{\prime}+\left|A_{j 2}^{\prime}\right| \boldsymbol{\tau}_{j k 2}^{\prime}\right) \cdot \mathbf{u}_{j}+\sum_{j \in E(k) \cap \Gamma} \frac{1}{2}\left|A_{j}\right| \mathbf{u}_{j} \cdot \boldsymbol{\tau}_{j}\right) .
\end{aligned}
$$

Remark that if the node $S_{k}$ is not on the boundary $\Gamma$ (i.e. if $k \notin \Gamma$ ), then the set $E(k) \cap \Gamma$ is empty. On the contrary, if $P_{k}$ is a boundary dual cell, then the set $E(k) \cap \Gamma$ is composed of the two boundary edges which have $S_{k}$ as a vertex. In this case, the quantity $\sum_{j \in E(k) \cap \Gamma} \frac{1}{2}\left|A_{j}\right| \mathbf{u}_{j} \cdot \mathbf{n}_{j}$ (resp. $\sum_{j \in E(k) \cap \Gamma} \frac{1}{2}\left|A_{j}\right| \mathbf{u}_{j} \cdot \boldsymbol{\tau}_{j}$ ) is an approximation of $\int_{\partial P_{k} \cap \Gamma} \mathbf{u} \cdot \mathbf{n}(\xi) d \xi\left(\right.$ resp. $\int_{\partial P_{k} \cap \Gamma} \mathbf{u} \cdot \boldsymbol{\tau}(\xi) d \xi$.) For a given vector field $\mathbf{u}$, it is straightforward to check that formulae (3.3) (resp. (3.4)) are the exact mean-values of $\nabla \cdot \mathbf{u}($ resp. $\nabla \cdot \boldsymbol{\tau})$ over $T_{i}$ and an inner $P_{k}$ if $\left|A_{j}\right| \mathbf{u}_{j} \cdot \mathbf{n}_{j i}=\int_{A_{j}} \mathbf{u} \cdot \mathbf{n}_{j i} d s$ (resp. $\left.\left|A_{j}\right| \mathbf{u}_{j} \cdot \boldsymbol{\tau}_{j i}=\int_{A_{j}} \mathbf{u} \cdot \boldsymbol{\tau}_{j i} d s\right)$ and $\left(\left|A_{j 1}^{\prime}\right| \mathbf{n}_{j k 1}^{\prime}+\left|A_{j 2}^{\prime}\right| \mathbf{n}_{j k 2}^{\prime}\right) \cdot \mathbf{u}_{j}=\int_{A_{j 1}^{\prime}} \mathbf{u} \cdot \mathbf{n}_{j k 1}^{\prime} d s+\int_{A_{j 2}^{\prime}} \mathbf{u} \cdot \mathbf{n}_{j k 2}^{\prime} d s$ (resp. $\left(\left|A_{j 1}^{\prime}\right| \boldsymbol{\tau}_{j k 1}^{\prime}+\left|A_{j 2}^{\prime}\right| \boldsymbol{\tau}_{j k 2}^{\prime}\right) \cdot \mathbf{u}_{j}=\int_{A_{j 1}^{\prime}} \mathbf{u} \cdot \boldsymbol{\tau}_{j k 1}^{\prime} d s+\int_{A_{j 2}^{\prime}} \mathbf{u} \cdot \boldsymbol{\tau}_{j k 2}^{\prime} d s$ ).

Now, we define discrete scalar products and a trace operator. 
Definition 3.3. For $(\phi, \psi) \in\left(\mathbb{R}^{I} \times \mathbb{R}^{K}\right)^{2}$ and $(\mathbf{u}, \mathbf{v}) \in\left(\left(\mathbb{R}^{2}\right)^{J}\right)^{2}$, we set:

$$
\begin{aligned}
(\mathbf{u}, \mathbf{v})_{D} & :=\sum_{j \in[1, J]}\left|D_{j}\right| \mathbf{u}_{j} \cdot \mathbf{v}_{j}, \\
(\phi, \psi)_{T, P} & :=\frac{1}{2}\left(\sum_{i \in[1, I]}\left|T_{i}\right| \phi_{i}^{T} \psi_{i}^{T}+\sum_{k \in[1, K]}\left|P_{k}\right| \phi_{k}^{P} \psi_{k}^{P}\right) .
\end{aligned}
$$

The associated norms will be denoted by $\|\cdot\|_{D}$ and $\|\cdot\|_{T, P}$. We also define discrete scalar products on the boundaries $\Gamma_{q}$ and $\Gamma$ : for sets of values $\left(u_{j}, v_{j}\right)_{j \in \Gamma}$ we set:

$$
(u, v)_{\Gamma_{q}, h}:=\sum_{j \in \Gamma_{q}}\left|A_{j}\right| u_{j} v_{j} \quad, \quad(u, v)_{\Gamma, h}:=\sum_{q \in[0, Q]}(u, v)_{\Gamma_{q}, h} .
$$

In particular, we shall often consider these boundary scalar products for the trace $\tilde{\phi}$ of a given $\phi \in \mathbb{R}^{I+J^{\Gamma}} \times \mathbb{R}^{K}$, defined by

Definition 3.4. (Trace operator) For any $\phi \in \mathbb{R}^{I+J^{\Gamma}} \times \mathbb{R}^{K}$, and for any $j \in \Gamma$, we set

$$
\tilde{\phi}_{j}:=\frac{1}{4}\left(\phi_{k_{1}(j)}^{P}+2 \phi_{i_{2}(j)}^{T}+\phi_{k_{2}(j)}^{P}\right) .
$$

With these scalar products, the discrete operators verify properties which are analogues to properties of the continuous operators $[25,15]$ :

Proposition 3.5. The following discrete analogues of the Green formulae hold:

$$
\begin{gathered}
\left(\nabla_{h}^{T, P} \cdot \mathbf{u}, \phi\right)_{T, P}=-\left(\mathbf{u}, \nabla_{h}^{D} \phi\right)_{D}+(\mathbf{u} \cdot \mathbf{n}, \tilde{\phi})_{\Gamma, h}, \\
\left(\nabla_{h}^{T, P} \times \mathbf{u}, \phi\right)_{T, P}=\left(\mathbf{u}, \nabla_{h}^{D} \times \phi\right)_{D}+(\mathbf{u} \cdot \boldsymbol{\tau}, \tilde{\phi})_{\Gamma, h},
\end{gathered}
$$

for all $\mathbf{u} \in\left(\mathbb{R}^{J}\right)^{2}$ and all $\phi=\left(\phi^{T}, \phi^{P}\right) \in \mathbb{R}^{I+J^{\Gamma}} \times \mathbb{R}^{K}$.

The following two propositions state discrete equivalents of the continuous properties $\nabla$. $(\boldsymbol{\nabla} \times)=0, \nabla \times \boldsymbol{\nabla}=0$ and $\nabla \times(\boldsymbol{\nabla} \times)=-\nabla \cdot \nabla$ (in two dimensions). Note however the hypothesis needed on the boundary dual cells:

Proposition 3.6. For all $\phi=\left(\phi^{T}, \phi^{P}\right) \in \mathbb{R}^{I+J^{\Gamma}} \times \mathbb{R}^{K}$, the following equalities hold:

$$
\begin{aligned}
& \left(\nabla_{h}^{T, P} \cdot\left(\nabla_{h}^{D} \times \phi\right)\right)_{i, k}=0, \quad \forall i \in[1, I], \forall k \notin \Gamma, \\
& \left(\nabla_{h}^{T, P} \times\left(\nabla_{h}^{D} \phi\right)\right)_{i, k}=0, \quad \forall i \in[1, I], \forall k \notin \Gamma .
\end{aligned}
$$

Moreover, these formulae still hold on boundary dual cells $P_{k}, k \in \Gamma$, if for any $q \in[0, Q]$, there exist two real numbers $\left(c_{q}^{T}, c_{q}^{P}\right)$ such that $\phi_{i}^{T}=c_{q}^{T}$ and $\phi_{k}^{P}=c_{q}^{P}$ uniformly over $\Gamma_{q}$.

Proposition 3.7. For all $\phi=\left(\phi^{T}, \phi^{P}\right) \in \mathbb{R}^{I+J^{\Gamma}} \times \mathbb{R}^{K}$, the following equality holds:

$$
\left(\nabla_{h}^{T, P} \times \nabla_{h}^{D} \times \phi\right)_{i, k}=-\left(\nabla_{h}^{T, P} \cdot \nabla_{h}^{D} \phi\right)_{i, k}, \quad \forall i \in[1, I], \forall k \in[1, K] .
$$


Finally, a discrete Helmholtz-Hodge decomposition of vector fields in non simply connected domains holds [25]:

Proposition 3.8. Let $\left(\mathbf{u}_{j}\right)_{j \in[1, J]}$ be a discrete vector field defined by its values on the diamond cells $D_{j}$. Then, there exist unique $\phi=\left(\phi_{i}^{T}, \phi_{k}^{P}\right)$ and $\psi=\left(\psi_{i}^{T}, \psi_{k}^{P}\right)$ both in $\mathbb{R}^{I+J^{\Gamma}} \times \mathbb{R}^{K}$ and $\left(c_{q}^{T}, c_{q}^{P}\right)$ in $\mathbb{R}^{Q} \times \mathbb{R}^{Q}$ such that:

$$
\begin{aligned}
& \mathbf{u}_{j}=\left(\nabla_{h}^{D} \phi\right)_{j}+\left(\nabla_{h}^{D} \times \psi\right)_{j}, \quad \forall j \in[1, J], \\
& \text { with } \sum_{i \in[1, I]}\left|T_{i}\right| \phi_{i}^{T}=\sum_{k \in[1, K]}\left|P_{k}\right| \phi_{k}^{P}=0, \\
& \forall q \in[1, Q], \quad \psi_{i}^{T}=0, \forall i \in \Gamma_{0} \quad, \quad \psi_{k}^{P}=0, \forall k \in \Gamma_{0}, \\
& \psi_{q}^{T}, \forall i \in \Gamma_{q} \quad, \quad \psi_{k}^{P}=c_{q}^{P}, \forall k \in \Gamma_{q} .
\end{aligned}
$$

Moreover, the decomposition (3.14) is orthogonal, in the sense that

$$
\left(\nabla_{h}^{D} \phi, \nabla_{h}^{D} \times \psi\right)_{D}=0,
$$

a property that is true for all $\phi$ and all $\psi$ verifying (3.16) and (3.17).

Formulae (3.15) are discrete analogues (respectively stated on the primal mesh and on the dual mesh) of the condition $\int_{\Omega} \phi=0$ that appears in the continuous Helmholtz-Hodge decomposition, while formulae (3.16) and (3.17) are discrete analogues of the boundary conditions $\psi=0$ on $\Gamma_{0}$ and $\psi=c_{q}$ on $\Gamma_{q}$.

Remark 3.9. We may also write a similar decomposition by changing the conditions (3.15)(3.17) in the following way

$$
\begin{aligned}
& \sum_{i \in[1, I]}\left|T_{i}\right| \psi_{i}^{T}=\sum_{k \in[1, K]}\left|P_{k}\right| \psi_{k}^{P}=0, \\
\phi_{i}^{T}= & 0, \forall i \in \Gamma_{0} \quad, \quad \phi_{k}^{P}=0, \forall k \in \Gamma_{0}, \\
\forall q \in[1, Q], \quad \phi_{i}^{T}= & c_{q}^{T}, \forall i \in \Gamma_{q}, \quad \phi_{k}^{P}=c_{q}^{P}, \forall k \in \Gamma_{q} .
\end{aligned}
$$

Finally, we mention discrete analogues of the so-called Poincaré inequalities, which were proved in [35] (respectively Theorem 3.2 and Theorem 3.6):

Proposition 3.10. Let $\phi=\left(\phi_{i}^{T}, \phi_{k}^{P}\right)$ and $\psi=\left(\psi_{i}^{T}, \psi_{k}^{P}\right)$ both in $\mathbb{R}^{I+J^{\Gamma}} \times \mathbb{R}^{K}$ and $\left(c_{q}^{T}, c_{q}^{P}\right)$ in $\mathbb{R}^{Q} \times \mathbb{R}^{Q}$ be such that (3.15), (3.16) and (3.17) hold; then, there exists a constant $C\left(\theta^{*}\right)$, depending only on $\theta^{*}$ and on $\Omega$ such that

$$
\|\phi\|_{T, P} \leq C\left(\theta^{*}\right)\left\|\nabla_{h}^{D} \phi\right\|_{D}, \quad\|\psi\|_{T, P} \leq C\left(\theta^{*}\right)\left\|\nabla_{h}^{D} \psi\right\|_{D} .
$$

\section{Application to the Stokes equations}

In this section, we are interested in the discretization of Eqs. (1.7), (1.8) and (1.2) supplemented with one of the following non-standard sets of conditions, which generalize conditions (1.3) to 
(1.6) to non-simply connected domains, with $\sigma, p_{d}$ and $\omega_{d}$ given functions, $m_{\omega}$ a given real number and $\left(k_{q}\right)_{q \in[1, Q]}$ a set of given real numbers:

$$
\begin{aligned}
& \left\{\begin{array}{l}
\mathbf{u} \cdot \mathbf{n}=\sigma \text { over } \Gamma \quad, \quad \omega_{\mid \Gamma_{0}}=\omega_{d} ; \quad \omega_{\mid \Gamma_{q}}=\omega_{d}+c_{q}, \forall q \in[1, Q], \\
\int_{\Gamma_{q}} \mathbf{u} \cdot \boldsymbol{\tau}=k_{q}, \forall q \in[1, Q], \quad \int_{\Omega} p(\mathbf{x}) d \mathbf{x}=0,
\end{array}\right. \\
& \left\{\begin{array}{l}
\mathbf{u} \cdot \mathbf{n}=\sigma \text { over } \Gamma, \quad p_{\mid \Gamma_{0}}=p_{d} ; \quad p_{\mid \Gamma_{q}}=p_{d}+c_{q}, \forall q \in[1, Q], \\
\int_{\Gamma_{q}} \mathbf{u} \cdot \boldsymbol{\tau}=k_{q}, \forall q \in[1, Q], \quad \int_{\Omega} \omega(\mathbf{x}) d \mathbf{x}=m_{\omega},
\end{array}\right. \\
& \left\{\begin{array}{l}
\mathbf{u} \cdot \boldsymbol{\tau}=\sigma \text { over } \Gamma, \quad \omega_{\mid \Gamma_{0}}=\omega_{d} ; \quad \omega_{\mid \Gamma_{q}}=\omega_{d}+c_{q}, \forall q \in[1, Q], \\
\int_{\Gamma_{q}} \mathbf{u} \cdot \mathbf{n}=k_{q}, \forall q \in[1, Q], \quad \int_{\Omega} p(\mathbf{x}) d \mathbf{x}=0,
\end{array}\right. \\
& \left\{\begin{array}{l}
\mathbf{u} \cdot \boldsymbol{\tau}=\sigma \text { over } \Gamma, \quad p_{\mid \Gamma_{0}}=p_{d} ; \quad p_{\mid \Gamma_{q}}=p_{d}+c_{q}, \forall q \in[1, Q], \\
\int_{\Gamma_{q}} \mathbf{u} \cdot \mathbf{n}=k_{q}, \forall q \in[1, Q], \quad \int_{\Omega} \omega(\mathbf{x}) d \mathbf{x}=m_{\omega},
\end{array}\right.
\end{aligned}
$$

where the constants $\left(c_{q}\right) \in \mathbb{R}^{Q}$ have to be determined.

Before going into details of the discretization of these equations, we discuss the compatibility conditions of the right-hand sides of these sets of equations.

\subsection{Compatibility conditions}

Consider Eqs. (1.2), (1.7) and (1.8). First, when associated with one of the sets of conditions (4.1) or (4.2), the data has to verify

$$
\int_{\Omega} g(\mathbf{x}) d \mathbf{x}=\int_{\Gamma} \sigma(\xi) d \xi .
$$

Then, when associated with the set (4.4), integration of (1.8) over $\Omega$ and application of the Green formula and of the boundary conditions yield

$$
\int_{\Gamma} \sigma(\xi) d \xi=m_{\omega}
$$

Finally, in the case of Eqs. (1.2), (1.7) and (1.8) associated with the set (4.3), the situation is more involved. Indeed, we infer from (1.8) that $\int_{\Gamma} \sigma(\xi) d \xi=\int_{\Omega} \omega(\mathbf{x}) d \mathbf{x}$. However, the quantity $\int_{\Omega} \omega(\mathbf{x}) d \mathbf{x}$ is not a data given by (4.3), but is a result of the computation of $\omega$ through the Hodge decomposition of $\mathbf{f}+\boldsymbol{\nabla} g$ expressed by Eq. (1.7) associated with the conditions over $p$ and $\omega$ expressed in the set (4.3). This may be interpreted as an implicit compatibility condition between the boundary conditions $\omega_{d}$ and $\sigma$ in (4.3). Further details on how to handle this will be given in subsection 4.2 .

\subsection{Discretization of the Stokes equations in vorticity-velocity-pressure formulation}

In this subsection, we are interested in the approximation of the continuous problem given by (1.2)-(1.7)-(1.8) associated with one of the sets of conditions (4.1) to (4.4). We choose to 
approach the solution of this problem by a vector $\left(\mathbf{u}_{j}\right)$, with $j \in[1, J]$, which discretizes the velocity field by values defined over the diamond cells of the mesh, and by scalars $\left(\omega_{i}^{T}, \omega_{k}^{P}\right)$ and $\left(p_{i}^{T}, p_{k}^{P}\right)$, with $i \in\left[1, I+J^{\Gamma}\right], k \in[1, K]$, which discretize the vorticity and the pressure fields by values defined over the primal and dual cells, as well as boundary edges of the mesh. The problem will be solved in two steps. In the first, we use the Hodge decomposition of $\mathbf{f}+\boldsymbol{\nabla} g$ (see prop. 3.8) to solve for $p$ and $\omega$. In the second, we solve a div-curl problem for $\mathbf{u}$.

Step 1: The discrete Hodge decomposition of the data $\mathbf{f}+\nabla g$ reads: find $p=\left(p_{i}^{T}, p_{k}^{P}\right)_{i \in\left[1, I+J^{\Gamma}\right], k \in[1, K]}, \omega=\left(\omega_{i}^{T}, \omega_{k}^{P}\right)_{i \in\left[1, I+J^{\Gamma}\right], k \in[1, K]}$ and $\left(c_{q}^{T}, c_{q}^{P}\right)_{q \in[1, Q]}$ such that

$$
\left(\nabla_{h}^{D} p\right)_{j}+\left(\nabla_{h}^{D} \times \omega\right)_{j}=\mathbf{f}_{j}^{D}+(\nabla g)_{j}^{D}, \quad \forall j \in[1, J],
$$

together with one of the following sets of conditions

$$
\left\{\begin{array}{l}
\omega_{i}^{T}=\omega_{d}\left(G_{i}\right), \forall i \in \Gamma_{0}, \quad \omega_{i}^{T}=\omega_{d}\left(G_{i}\right)+c_{q}^{T}, \forall i \in \Gamma_{q}, \forall q, \\
\omega_{k}^{P}=\omega_{d}\left(S_{k}\right), \forall k \in \Gamma_{0}, \quad \omega_{k}^{P}=\omega_{d}\left(S_{k}\right)+c_{q}^{P}, \forall k \in \Gamma_{q}, \forall q, \\
\sum_{i \in[1, I]}\left|T_{i}\right| p_{i}^{T}=\sum_{k \in[1, K]}\left|P_{k}\right| p_{k}^{P}=0,
\end{array}\right.
$$

for a given (up to constants to be determined on each internal boundary) vorticity field $\omega_{d}$ on the boundary (see the corresponding equations in (4.1) and (4.3)), or

$$
\left\{\begin{array}{l}
p_{i}^{T}=p_{d}\left(G_{i}\right), \forall i \in \Gamma_{0}, \quad p_{i}^{T}=p_{d}\left(G_{i}\right)+c_{q}^{T}, \forall i \in \Gamma_{q}, \forall q, \\
p_{k}^{P}=p_{d}\left(S_{k}\right), \forall k \in \Gamma_{0}, \quad p_{k}^{P}=p_{d}\left(S_{k}\right)+c_{q}^{P}, \forall k \in \Gamma_{q}, \forall q \\
\sum_{i \in[1, I]}\left|T_{i}\right| \omega_{i}^{T}=\sum_{k \in[1, K]}\left|P_{k}\right| \omega_{k}^{P}=m_{\omega},
\end{array}\right.
$$

for a given (up to constants to be determined on each internal boundary) pressure field $p_{d}$ on the boundary (see the corresponding equations in (4.2) and (4.4)).

In (4.7), the following definitions have been used

$$
\mathbf{f}_{j}^{D}=\frac{1}{\left|D_{j}\right|} \int_{D_{j}} \mathbf{f}(\mathbf{x}) d \mathbf{x} \quad, \quad(\nabla g)_{j}^{D}=\frac{1}{\left|D_{j}\right|} \int_{D_{j}} \nabla g(\mathbf{x}) d \mathbf{x} \quad \forall j \in[1, J] .
$$

The two problems involving (4.7) and (4.8) on the one hand, and (4.7) and (4.9) on the other hand are solved in a very similar way, thus we only detail the solution of (4.7)-(4.8).

Proposition 4.1. Problem (4.7)-(4.8) may be split into two independent subproblems: setting $\mathbf{s}:=\left(\mathbf{s}_{j}^{D}\right)_{j \in[1, J]}$ with $\mathbf{s}_{j}^{D}:=\mathbf{f}_{j}^{D}+(\nabla g)_{j}^{D}$, find $\left(\omega_{i}^{T}, \omega_{k}^{P}\right)_{i \in\left[1, I+J^{\Gamma}\right], k \in[1, K]}$ and $\left(c_{q}^{T}, c_{q}^{P}\right)_{q \in[1, Q]}$ such that

$$
\left\{\begin{aligned}
-\left(\nabla_{h}^{T} \cdot \nabla_{h}^{D} \omega\right)_{i} & =\left(\nabla_{h}^{T} \times \mathbf{s}\right)_{i}, \quad \forall i \in[1, I], \\
-\left(\nabla_{h}^{P} \cdot \nabla_{h}^{D} \omega\right)_{k} & =\left(\nabla_{h}^{P} \times \mathbf{s}\right)_{k}, \quad \forall k \notin \Gamma, \\
\left(\nabla_{h}^{D} \omega \cdot \mathbf{n}, 1\right)_{\Gamma_{q}, h} & =-(\mathbf{s} \cdot \boldsymbol{\tau}, 1)_{\Gamma_{q}, h}, \quad \forall q \in[1, Q], \\
-\sum_{k \in \Gamma_{q}}\left|P_{k}\right|\left(\nabla_{h}^{P} \cdot \nabla_{h}^{D} \omega\right)_{k} & =\sum_{k \in \Gamma_{q}}\left|P_{k}\right|\left(\nabla_{h}^{P} \times \mathbf{s}\right)_{k}, \quad \forall q \in[1, Q], \\
\omega_{i}^{T}=\omega_{d}\left(G_{i}\right), \quad \forall i \in \Gamma_{0} & , \quad \omega_{k}^{P}=\omega_{d}\left(S_{k}\right), \quad \forall k \in \Gamma_{0}, \\
\omega_{i}^{T}=\omega_{d}\left(G_{i}\right)+c_{q}^{T}, \quad \forall i \in \Gamma_{q} & , \quad \omega_{k}^{P}=\omega_{d}\left(S_{k}\right)+c_{q}^{P}, \quad \forall k \in \Gamma_{q}, \quad \forall q \in[1, Q],
\end{aligned}\right.
$$


and, once $\omega$ has been computed, find $\left(p_{i}^{T}, p_{k}^{P}\right)_{i \in\left[1, I+J^{\Gamma}\right], k \in[1, K]}$ such that

$$
\left\{\begin{aligned}
\left(\nabla_{h}^{T} \cdot \nabla_{h}^{D} p\right)_{i} & =\left(\nabla_{h}^{T} \cdot \mathbf{s}\right)_{i}, \quad \forall i \in[1, I], \\
\left(\nabla_{h}^{P} \cdot \nabla_{h}^{D} p\right)_{k} & =\left(\nabla_{h}^{P} \cdot\left(\mathbf{s}-\nabla_{h}^{D} \times \omega\right)\right)_{k}, \forall k \in[1, K], \\
\left(\boldsymbol{\nabla}_{h}^{D} p\right)_{j} \cdot \mathbf{n}_{j} & \left.=\left(\mathbf{s}_{j}-\left(\boldsymbol{\nabla}_{h}^{D} \times \omega\right)_{j}\right)\right) \cdot \mathbf{n}_{j}, \quad \forall j \in \Gamma, \\
\sum_{i \in[1, I]}\left|T_{i}\right| p_{i}^{T} & =\sum_{k \in[1, K]}\left|P_{k}\right| p_{k}^{P}=0 .
\end{aligned}\right.
$$

Proof. Applying the discrete vector curl operator to (4.7) on any primal cell and on any inner dual cell yields the first two lines of (4.11), thanks to (3.12) and (3.13).

Next, for a given $q \in[1, Q]$, we consider the element $\psi \in \mathbb{R}^{I+J^{\Gamma}} \times \mathbb{R}^{K}$ which has the following values: $\psi_{i}^{T}=1, \forall i \in \Gamma_{q}$ and $\psi_{i}^{T}=0$ everywhere else, and $\psi_{k}^{P}=0$ everywhere. Then, we compute the scalar product (3.5) of Eq. (4.7) with $\nabla_{h}^{D} \times \psi$ :

$$
\left(\nabla_{h}^{D} \times \omega, \nabla_{h}^{D} \times \psi\right)_{D}+\left(\nabla_{h}^{D} p, \nabla_{h}^{D} \times \psi\right)_{D}=\left(\mathbf{s}, \nabla_{h}^{D} \times \psi\right)_{D} .
$$

Using the orthogonality of $\boldsymbol{\nabla}_{h}^{D} p$ and $\nabla_{h}^{D} \times \psi$ (see last line of Prop. 3.8), using the discrete Green formula (3.10) and Eq. (3.13), we infer

$$
-\left(\nabla_{h}^{T, P} \cdot \nabla_{h}^{D} \omega, \psi\right)_{T, P}-\left(\nabla_{h}^{D} \times \omega \cdot \boldsymbol{\tau}, \tilde{\psi}\right)_{\Gamma, h}=\left(\nabla_{h}^{T, P} \times \mathbf{s}, \psi\right)_{T, P}-(\mathbf{s} \cdot \boldsymbol{\tau}, \tilde{\psi})_{\Gamma, h} .
$$

Further, using the fact that $\psi$ vanishes everywhere but on the boundary points $G_{i}$, the first term in the left-hand side and the first term in the right-hand side of (4.14) vanish. Next, since $\boldsymbol{\nabla}_{h}^{D} \times \omega \cdot \boldsymbol{\tau}=-\boldsymbol{\nabla}_{h}^{D} \omega \cdot \mathbf{n}$, and using the definition (3.7) and the fact that the values of $\psi$ on the boundaries imply that $\tilde{\psi}_{j}=1 / 2$ for all $j \in \Gamma_{q}$ and $\tilde{\psi}_{j}=0$ for all $j \in \Gamma_{q}^{\prime}$ for any $q^{\prime} \neq q$, Equ. (4.14) implies $-\left(\nabla_{h}^{D} \omega \cdot \mathbf{n}, \frac{1}{2}\right)_{\Gamma_{q}, h}=\left(\mathbf{s} \cdot \boldsymbol{\tau}, \frac{1}{2}\right)_{\Gamma_{q}, h}$ and thus the third line of (4.11).

Last, we consider the element $\psi \in \mathbb{R}^{I+J^{\Gamma}} \times \mathbb{R}^{K}$ which has the following values: $\psi_{i}^{T}=1$, $\forall i \in \Gamma_{q}$ and $\psi_{k}^{P}=1, \forall k \in \Gamma_{q}$ and vanishing everywhere else. Then, the dot product of Eq. (4.7) with $\nabla_{h}^{D} \times \psi$ yields (4.13) and (4.14) again. Now, we use the fact that the first term in the left-hand side of (4.14) equals $-\frac{1}{2} \sum_{k \in \Gamma_{q}}\left|P_{k}\right|\left(\nabla_{h}^{P} \cdot \nabla_{h}^{D} \omega\right)_{k}$ while the first term in the right-hand side of (4.14) equals $\frac{1}{2} \sum_{k \in \Gamma_{q}}\left|P_{k}\right|\left(\nabla_{h}^{P} \times \mathbf{s}\right)_{k}$. Using the previously proved third line of (4.11), we obtain the fourth line of (4.11).

Once $\omega$ has been computed, the derivation of (4.12) from (4.7)-(4.8) is obvious. We stress that in the second equation of (4.12), there holds $\left(\nabla_{h}^{P} \cdot \nabla_{h}^{D} \times \omega\right)_{k}=0$ for all inner dual cells $(k \notin \Gamma)$ but that this property might not necessarily be true for boundary dual cells $(k \in \Gamma)$, see Prop. 3.6.

Step 1 ends with the fact that (4.11) and (4.12) are well-posed:

Lemma 4.2. It was shown in [25, Proposition 5.2] that systems of types (4.11) and (4.12) both have a unique solution.

Now, let us describe Step 2 of the calculations, which consists in solving for the velocity. 
Step 2: Once $\left(\omega_{i}^{T}, \omega_{k}^{P}\right)_{i \in[1, I], k \in[1, K]}$ has been computed through Step 1, we solve a div-curl problem for $\mathbf{u}$ : given $\left(k_{q}\right)_{q \in[1, Q]}$, find $\left(\mathbf{u}_{j}\right)_{j \in[1, J]}$ such that

$$
\left\{\begin{array}{rlrl}
\left(\nabla_{h}^{T, P} \cdot \mathbf{u}\right)_{i, k} & =g_{i, k}^{T, P}, & & \forall i \in[1, I], \forall k \in[1, K], \\
\left(\nabla_{h}^{T, P} \times \mathbf{u}\right)_{i, k} & =\omega_{i, k}^{T, P}, & \forall i \in[1, I], \forall k \notin \Gamma, \\
\mathbf{u}_{j} \cdot \mathbf{n}_{j} & =\sigma_{j}, & \forall j \in \Gamma, \\
(\mathbf{u} \cdot \boldsymbol{\tau}, 1)_{\Gamma_{q}, h} & =k_{q}, & \forall q \in[1, Q], \\
\sum_{k \in \Gamma_{q}}\left|P_{k}\right|\left(\nabla_{h}^{P} \times \mathbf{u}\right)_{k} & =\sum_{k \in \Gamma_{q}}\left|P_{k}\right| \omega_{k}^{P}, \quad \forall q \in[1, Q],
\end{array}\right.
$$

in the case of a given normal velocity field on the boundary (Eqs. (4.1) or (4.2)) or

$$
\left\{\begin{array}{rlrl}
\left(\nabla_{h}^{T, P} \cdot \mathbf{u}\right)_{i, k} & =g_{i, k}^{T, P}, & & \forall i \in[1, I], \forall k \notin \Gamma, \\
\left(\nabla_{h}^{T, P} \times \mathbf{u}\right)_{i, k} & =\omega_{i, P}^{T, P}, & & \forall i \in[1, I], \forall k \in[1, K], \\
\mathbf{u}_{j} \cdot \boldsymbol{\tau}_{j} & =\sigma_{j}, & & \forall j \in \Gamma, \\
(\mathbf{u} \cdot \mathbf{n}, 1)_{\Gamma_{q}, h} & =k_{q}, & & \forall q \in[1, Q], \\
\sum_{k \in \Gamma_{q}}\left|P_{k}\right|\left(\nabla_{h}^{P} \cdot \mathbf{u}\right)_{k} & =\sum_{k \in \Gamma_{q}} & \left|P_{k}\right| g_{k}^{P}, \quad \forall q \in[1, Q],
\end{array}\right.
$$

in the case of a given tangential velocity field on the boundary (Eqs. (4.3) or (4.4)).

In (4.15) and (4.16), we have set

$$
\begin{gathered}
g_{i}^{T}=\frac{1}{\left|T_{i}\right|} \int_{T_{i}} g(\mathbf{x}) d \mathbf{x} \quad \forall i \in[1, I] \quad, \quad g_{k}^{P}=\frac{1}{\left|P_{k}\right|} \int_{P_{k}} g(\mathbf{x}) d \mathbf{x} \quad \forall k \in[1, K], \\
\sigma_{j}=\frac{1}{\left|A_{j}\right|} \int_{A_{j}} \sigma(\xi) d \xi \quad \forall j \in \Gamma .
\end{gathered}
$$

Moreover, the right-hand sides in (4.15) and (4.16) have to verify compatibility conditions. Indeed, it is readily seen from the definition of the discrete divergence and curl operators (3.3) and (3.4) that the following two equalities hold

$$
\begin{aligned}
\sum_{i \in[1, I]}\left|T_{i}\right|\left(\nabla_{h}^{T} \cdot \mathbf{u}\right)_{i} & =\sum_{k \in[1, K]}\left|P_{k}\right|\left(\nabla_{h}^{P} \cdot \mathbf{u}\right)_{k}=\sum_{j \in \Gamma}\left|A_{j}\right| \mathbf{u}_{j} \cdot \mathbf{n}_{j} \\
\sum_{i \in[1, I]}\left|T_{i}\right|\left(\nabla_{h}^{T} \times \mathbf{u}\right)_{i} & =\sum_{k \in[1, K]}\left|P_{k}\right|\left(\nabla_{h}^{P} \times \mathbf{u}\right)_{k}=\sum_{j \in \Gamma}\left|A_{j}\right| \mathbf{u}_{j} \cdot \boldsymbol{\tau}_{j}
\end{aligned}
$$

Then, because of (4.19), the right-hand sides in (4.15) must satisfy

$$
\sum_{i \in[1, I]}\left|T_{i}\right| g_{i}^{T}=\sum_{k \in[1, K]}\left|P_{k}\right| g_{k}^{P}=\sum_{j \in \Gamma}\left|A_{j}\right| \sigma_{j} .
$$

This relation is true thanks to the definitions (4.17) and (4.18) since

$$
\sum_{i \in[1, I]}\left|T_{i}\right| g_{i}^{T}=\sum_{k \in[1, K]}\left|P_{k}\right| g_{k}^{P}=\int_{\Omega} g(\mathbf{x}) d \mathbf{x} \quad \text { and } \quad \sum_{j \in \Gamma}\left|A_{j}\right| \sigma_{j}=\int_{\Gamma} \sigma(\xi) d \xi
$$


That the right-hand sides of the above two equalities are identical follows from (4.5).

Further, because of (4.20), the right-hand sides in (4.16) must satisfy

$$
\sum_{i \in[1, I]}\left|T_{i}\right| \omega_{i}^{T}=\sum_{k \in[1, K]}\left|P_{k}\right| \omega_{k}^{P}=\sum_{j \in \Gamma}\left|A_{j}\right| \sigma_{j} .
$$

For the set of conditions (4.9), the first two terms in (4.22) are equal to $m_{\omega}$ and the last term in (4.22) is equal to $\int_{\Gamma} \sigma(\xi) d \xi$. These two quantities are identical thanks to (4.6).

On the other hand, for the set of conditions (4.8), the values of the first two terms in (4.22) are never imposed, but, rather, are results of the computations involved in the first step of our procedure, see Eq. (4.11), so that the compatibility condition (4.22) may not be verified in general. A possible way to overcome this problem is to change $\omega_{i}^{T}$ into $\omega_{i}^{T}+c^{T}$ and $\omega_{k}^{P}$ into $\omega_{k}^{P}+c^{P}$ for all $i \in\left[1, I+J^{\Gamma}\right]$ and all $k \in[1, K]$ in (4.16), with two constants $c^{T}$ and $c^{P}$ computed so that (4.22) holds. Doing so does not change the value of $\nabla_{h}^{D} \omega$, so that the modified $\omega$ still verifies system (4.11), but with modified boundary conditions. This may be interpreted as an implicit compatibility condition between the boundary conditions $\omega_{d}$ and $\sigma$ in (4.3).

Now, using the discrete Hodge decomposition of $\left(\mathbf{u}_{j}\right)_{j \in[1, J]}$, each of the problems (4.15) and (4.16) may be split into two independent subproblems involving the potentials. We only detail the resulting systems for problem (4.15) using the Hodge decomposition with boundary conditions (3.15) to (3.17). A similar result holds for problem (4.16) using the Hodge decomposition with boundary conditions (3.19) to (3.21).

Proposition 4.3. Problem (4.15) may be split into two independent problems: find $\left(\phi_{i}^{T}, \phi_{k}^{P}\right)_{i \in\left[1, I+J^{\Gamma}\right], k \in[1, K]}$ such that

$$
\left\{\begin{aligned}
\left(\nabla_{h}^{T} \cdot \nabla_{h}^{D} \phi\right)_{i} & =g_{i}^{T}, \quad \forall i \in[1, I], \\
\left(\nabla_{h}^{P} \cdot \nabla_{h}^{D} \phi\right)_{k} & =g_{k}^{P}, \quad \forall k \in[1, K], \\
\left(\nabla_{h}^{D} \phi\right)_{j} \cdot \mathbf{n}_{j} & =\sigma_{j}, \quad \forall j \in \Gamma, \\
\sum_{i \in[1, I]}\left|T_{i}\right| \phi_{i}^{T} & =\sum_{k \in[1, K]}\left|P_{k}\right| \phi_{k}^{P}=0,
\end{aligned}\right.
$$

and find $\left(\psi_{i}^{T}, \psi_{k}^{P}\right)_{i \in\left[1, I+J^{\Gamma}\right], k \in[1, K]}$ and $\left(c_{q}^{T}, c_{q}^{P}\right)_{q \in[1, Q]}$ such that

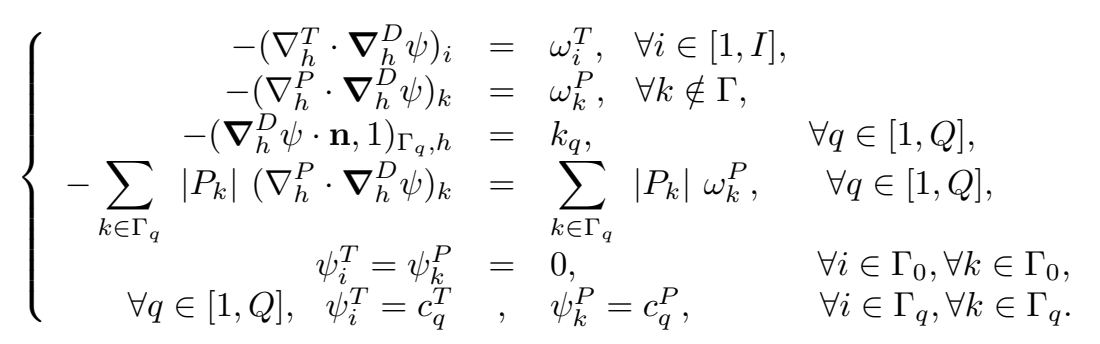

Proof. The proof is given in [25, Proposition 5.1].

Step 2 ends with the fact that (4.23) and (4.24) are well-posed:

Lemma 4.4. It was shown in [25, Proposition 5.2] that systems of the type (4.23) and (4.24) both have a unique solution. 
Once these two subproblems have been solved, the vector $\mathbf{u}$ is then reconstructed by $\mathbf{u}_{j}=$ $\left(\nabla_{h}^{D} \phi\right)_{j}+\left(\nabla_{h}^{D} \times \psi\right)_{j}$.

\section{$5 \quad$ Error estimates}

Obtaining error estimates usually relies on regularity assumptions on the solution of the problem. In order to apply results given in $[25,15]$, we shall assume more regularity on the vorticity and pressure fields $(\hat{\omega}, \hat{p})$ given by Proposition 5.1 below, and on the velocity potentials given by Proposition 5.3 below.

Proposition 5.1. Let $\left(\mathbf{f}, g, \sigma, \omega_{d}\right)$ belong to $L^{2}(\Omega)^{2} \times H^{1}(\Omega) \times H^{1 / 2}(\Gamma) \times H^{1 / 2}(\Gamma)$, and let $\left(k_{q}\right)_{q \in[1, Q]}$ be a set of given real numbers. Then, system (1.7)-(1.8)-(1.2)-(4.1) associated with (4.5) may be split into two subproblems, where $(\hat{p}, \hat{w}) \in H^{1}(\Omega)^{2}$ and a set of real numbers $\left(C_{q}\right)_{q \in[1, Q]}$ are the exact solution of the Hodge decomposition of $\mathbf{f}+\nabla g$ :

$$
\left\{\begin{aligned}
\nabla \times \hat{\omega}+\nabla \hat{p} & =\mathbf{f}+\nabla g \text { in } \Omega, \\
\hat{\omega}_{\mid \Gamma_{0}}=\omega_{d} ; & \hat{\omega}_{\mid \Gamma_{q}}=\omega_{d}+C_{q} \forall q \in[1, Q], \\
\int_{\Omega} \hat{p}(x) d x & =0,
\end{aligned}\right.
$$

and, once $\hat{\omega}$ has been determined, $\hat{\mathbf{u}} \in \operatorname{Hdiv}(\Omega) \cap H \operatorname{curl}(\Omega)$ solves the div-curl problem:

$$
\left\{\begin{aligned}
\nabla \cdot \hat{\mathbf{u}} & =g \text { in } \Omega, \\
\nabla \times \hat{\mathbf{u}} & =\hat{\omega} \text { in } \Omega, \\
\hat{\mathbf{u}} \cdot \mathbf{n} & =\sigma \text { on } \Gamma \\
\int_{\Gamma_{q}} \hat{\mathbf{u}} \cdot \boldsymbol{\tau} & =k_{q}, \forall q \in[1, Q] .
\end{aligned}\right.
$$

Hypothesis 5.2. We assume that $\hat{\omega}$ and $\hat{p}$ given by Proposition 5.1 belong to $H^{2}(\Omega)$.

The velocity field $\hat{\mathbf{u}}$ solving (5.2) may be found by the following Hodge decomposition.

Proposition 5.3. Let $(g, \hat{\omega}, \sigma)$ belong to $H^{1}(\Omega) \times H^{2}(\Omega) \times H^{1 / 2}(\Gamma)$, and let $\left(k_{q}\right)_{q \in[1, Q]}$ be a set of given real numbers; let $\hat{\mathbf{u}}$ be the exact solution of problem (5.2). Then, there exist $\hat{\phi}$ and $\hat{\psi}$ both in $H^{1}(\Omega)$ and a set of real numbers $\left(C_{q}\right)_{q \in[1, Q]}$ such that

$$
\hat{\mathbf{u}}=\nabla \hat{\phi}+\nabla \times \hat{\psi}
$$

where $\hat{\phi}$ and $\hat{\psi}$ are the solutions of

$$
\begin{aligned}
& \left\{\begin{array}{l}
\Delta \hat{\phi}=\nabla \cdot \hat{\mathbf{u}}=g \text { in } \Omega, \\
\boldsymbol{\nabla} \hat{\phi} \cdot \mathbf{n}=\hat{\mathbf{u}} \cdot \mathbf{n}=\sigma \text { on } \Gamma, \\
\int_{\Omega} \hat{\phi}=0,
\end{array}\right. \\
& \left\{\begin{array}{l}
-\Delta \hat{\psi}=\nabla \times \hat{\mathbf{u}}=\hat{\omega} \text { in } \Omega, \\
\hat{\psi}_{\mid \Gamma_{0}}=0 ; \hat{\psi}_{\mid \Gamma_{q}}=C_{q} \\
\int_{\Gamma_{q}} \nabla q \in[1, Q],
\end{array}\right.
\end{aligned}
$$


Proof. The Hodge decomposition of $\hat{\mathbf{u}}$ and the determination of $\hat{\phi}$ and $\hat{\psi}$ through (5.3) and (5.4) are direct consequences of [39, Theorem 3.2 and Corollary 3.1].

Hypothesis 5.4. We suppose that $\hat{\phi}$ and $\hat{\psi}$ given by Proposition 5.3 belong to $H^{2}(\Omega)$.

Due to re-entrant corners related to the internal polygonal boundaries $\Gamma_{q}$, the $H^{2}$ regularity of the potentials is not a consequence of the regularity of the data $(g, \hat{\omega}, \sigma)$.

\subsection{Error estimates in the $H^{1}$ semi-norm for the pressure and the vorticity}

Definition 5.5. For any continuous function $v$, we define $\Pi v$ by

$$
\begin{aligned}
\forall i \in\left[1, I+J^{\Gamma}\right], & & (\Pi v)_{i}^{T}=v\left(G_{i}\right), \\
\forall k \in[1, K], & & (\Pi v)_{k}^{P}=v\left(S_{k}\right) .
\end{aligned}
$$

We note that, under Hypothesis 5.2, $\hat{\omega}$ and $\hat{p}$ belong to $H^{2}(\Omega)$, which implies they are continuous on $\bar{\Omega}$, so that considering $\Pi \hat{\omega}$ and $\Pi \hat{p}$ makes sense.

Theorem 5.6. Let $p$ and $\omega$ be the solutions of the numerical scheme (4.7)-(4.8), and let $(\hat{p}, \hat{\omega})$ be the exact solutions of (5.1). Then, if all diamond-cells are convex and under assumptions 2.1 and 5.2, there exists a constant $C\left(\theta^{*}\right)$, independent of $h$, such that

$$
\left\|\nabla_{h}^{D}(p-\Pi \hat{p})\right\|_{D}+\left\|\nabla_{h}^{D}(\omega-\Pi \hat{\omega})\right\|_{D} \leq C\left(\theta^{*}\right) h\left(\|\hat{p}\|_{2, \Omega}+\|\hat{\omega}\|_{2, \Omega}\right) .
$$

Proof. Firstly, taking the mean-value of each term of the first line of Equ. (5.1) on a diamond cell $D_{j}$ and using (4.10), we get:

$$
\frac{1}{\left|D_{j}\right|} \int_{D_{j}}(\nabla \hat{p}+\nabla \times \hat{\omega})(\mathbf{x}) d \mathbf{x}=\mathbf{f}_{j}^{D}+(\nabla g)_{j}^{D}, \forall j \in[1, J] .
$$

Then, since (5.6) and (4.7) have the same right-hand side, we infer that:

$$
\left(\nabla_{h}^{D} p\right)_{j}+\left(\nabla_{h}^{D} \times \omega\right)_{j}=\frac{1}{\left|D_{j}\right|} \int_{D_{j}}(\nabla \hat{p}+\nabla \times \hat{\omega})(\mathbf{x}) d \mathbf{x}, \forall j \in[1, J] .
$$

Setting

$$
\varepsilon_{p}:=p-\Pi \hat{p} \text { and } \varepsilon_{\omega}:=\omega-\Pi \hat{\omega},
$$

Equ. (5.7) implies the following equality:

$$
\begin{array}{r}
\left(\nabla_{h}^{D} \varepsilon_{p}\right)_{j}+\left(\nabla_{h}^{D} \times \varepsilon_{\omega}\right)_{j}=\frac{1}{\left|D_{j}\right|} \int_{D_{j}}\left(\boldsymbol{\nabla} \hat{p}(\mathbf{x})-\left(\nabla_{h}^{D} \Pi \hat{p}\right)_{j}\right) d \mathbf{x} \\
+\frac{1}{\left|D_{j}\right|} \int_{D_{j}}\left(\nabla \times \hat{\omega}(\mathbf{x})-\left(\nabla_{h}^{D} \times \Pi \hat{\omega}\right)_{j}\right) d \mathbf{x}, \quad \forall j \in[1, J] .
\end{array}
$$

According to (4.8) and the second line of (5.1), the error $\varepsilon_{\omega}$ satisfies on the boundary

$$
\left\{\begin{array}{cl}
\left(\varepsilon_{\omega}\right)_{i}^{T}=0, \forall i \in \Gamma_{0} & , \quad\left(\varepsilon_{\omega}\right)_{i}^{T}=c_{q}^{T}-C_{q}, \quad \forall i \in \Gamma_{q}, \forall q \\
\left(\varepsilon_{\omega}\right)_{k}^{P}=0, \forall k \in \Gamma_{0} & , \quad\left(\varepsilon_{\omega}\right)_{k}^{P}=c_{q}^{P}-C_{q}, \quad \forall k \in \Gamma_{q}, \forall q .
\end{array}\right.
$$


Therefore, $\left(\nabla_{h}^{D} \varepsilon_{p}\right)$ and $\left(\nabla_{h}^{D} \times \varepsilon_{\omega}\right)$ are orthogonal for the scalar product $(\cdot, \cdot)_{D}$, as recalled at the end of Proposition 3.8. Consequently, multiplying (5.9) by $\left|D_{j}\right|\left(\nabla_{h}^{D} \varepsilon_{p}\right)_{j}$ and summing for all $j \in[1, J]$, we obtain:

$$
\begin{aligned}
\left\|\nabla_{h}^{D} \varepsilon_{p}\right\|_{D}^{2} & =\sum_{j \in[1, J]} \int_{D_{j}}\left(\nabla \hat{p}(\mathbf{x})-\left(\nabla_{h}^{D} \Pi \hat{p}\right)_{j}\right) \cdot\left(\nabla_{h}^{D} \varepsilon_{p}\right)_{j} d \mathbf{x} \\
& +\sum_{j \in[1, J]} \int_{D_{j}}\left(\boldsymbol{\nabla} \times \hat{\omega}(\mathbf{x})-\left(\nabla_{h}^{D} \times \Pi \hat{\omega}\right)_{j}\right) \cdot\left(\nabla_{h}^{D} \varepsilon_{p}\right)_{j} d \mathbf{x} .
\end{aligned}
$$

In the very same way, multiplying (5.9) by $\left|D_{j}\right|\left(\nabla_{h}^{D} \times \varepsilon_{\omega}\right)_{j}$ and summing for all $j \in[1, J]$, we obtain:

$$
\begin{aligned}
\left\|\nabla_{h}^{D} \varepsilon_{\omega}\right\|_{D}^{2} & =\sum_{j \in[1, J]} \int_{D_{j}}\left(\nabla \hat{p}(\mathbf{x})-\left(\nabla_{h}^{D} \Pi \hat{p}\right)_{j}\right) \cdot\left(\nabla_{h}^{D} \times \varepsilon_{\omega}\right)_{j} d \mathbf{x} \\
& +\sum_{j \in[1, J]} \int_{D_{j}}\left(\nabla \times \hat{\omega}(\mathbf{x})-\left(\nabla_{h}^{D} \times \Pi \hat{\omega}\right)_{j}\right) \cdot\left(\nabla_{h}^{D} \times \varepsilon_{\omega}\right)_{j} d \mathbf{x} .
\end{aligned}
$$

The right-hand sides of (5.11) and (5.12) may be bounded using the traditional $P^{1}$ Lagrange interpolations of $\hat{p}$ and $\hat{\omega}$ on a submesh of the diamond mesh, obtained by splitting each diamond cell, along any of its diagonals, into two triangles. The details of the calculations may be found in [15], starting with inequality (62) of that reference, and then using Lemma 5.11, where the norm in the right-hand side of inequality (66) has to be replaced by the $H^{2}(\Omega)$ norm of $\hat{p}$ and $\hat{\omega}$.

\subsection{Error estimates in the $L^{2}$-norm for the pressure and the vorticity}

We shall use the discrete Poincaré inequalities recalled in Proposition 3.10 to infer error estimates for the discrete $L^{2}$ norm of the errors in the pressure and the vorticity.

On the one hand, Proposition 3.10 can be applied directly to the error $\varepsilon_{\omega}$ because it verifies (5.10), which are exactly conditions (3.16) and (3.17) that Proposition 3.10 requires. Thus, we infer from (5.5) the following theorem

Theorem 5.7. There exists a constant $C\left(\theta^{*}\right)$ that does not depend on $h$ such that

$$
\|\omega-\Pi \hat{\omega}\|_{T, P} \leq C\left(\theta^{*}\right) h\left(\|\hat{p}\|_{2, \Omega}+\|\hat{\omega}\|_{2, \Omega}\right) .
$$

On the other hand, since $(p-\Pi \hat{p})$ does not in general verify the vanishing mean-value condition (3.15) (because $\Pi \hat{p}$ doesn't), we may not apply the discrete Poincaré inequality in a straightforward way. However, defining

$$
\begin{array}{r}
c^{T}:=\frac{\sum_{i \in[1, I]}\left|T_{i}\right|(\Pi \hat{p})_{i}^{T}}{|\Omega|}, \quad(\tilde{\Pi} \hat{p})_{i}^{T}:=(\Pi \hat{p})_{i}^{T}-c^{T}, \forall i \in\left[1, I+J^{\Gamma}\right], \\
c^{P}:=\frac{\sum_{k \in[1, K]}\left|P_{k}\right|(\Pi \hat{p})_{k}^{P}}{|\Omega|}, \quad(\tilde{\Pi} \hat{p})_{k}^{P}:=(\Pi \hat{p})_{k}^{P}-c^{P}, \forall k \in[1, K],
\end{array}
$$


we have that $\tilde{\Pi} p$ verifies the vanishing mean-value condition (3.15) and

$$
\nabla_{h}^{D}(\tilde{\Pi} \hat{p})=\nabla_{h}^{D}(\Pi \hat{p})
$$

because $(\tilde{\Pi} \hat{p})$ and $(\Pi \hat{p})$ only differ by a constant on the primal and dual meshes, and definition (3.1) of the discrete gradient implies that this does not affect its values. Further,

$$
\|p-\Pi \hat{p}\|_{T, P} \leq\|p-\tilde{\Pi} \hat{p}\|_{T, P}+\|\Pi \hat{p}-\tilde{\Pi} \hat{p}\|_{T, P} .
$$

Estimating the first term in the right-hand-side of (5.17) may be performed through the discrete Poincaré inequality applied to $(p-\tilde{\Pi} \hat{p})$.

Lemma 5.8. There exists a constant $C\left(\theta^{*}\right)$ that does not depend on $h$ such that

$$
\|p-\tilde{\Pi} \hat{p}\|_{T, P} \leq C\left(\theta^{*}\right) h\left(\|\hat{p}\|_{2, \Omega}+\|\hat{\omega}\|_{2, \Omega}\right) .
$$

Proof. From Prop. 3.10, it holds that

$$
\|p-\tilde{\Pi} \hat{p}\|_{T, P} \leq C\left(\theta^{*}\right)|| \nabla_{h}^{D}(p-\tilde{\Pi} \hat{p})\left\|_{D}=C\left(\theta^{*}\right)|| \nabla_{h}^{D}(p-\Pi \hat{p})\right\|_{D}
$$

because of (5.16). Applying (5.5), we get (5.18).

To estimate the second term in the right-hand-side of (5.17), we first recall (5.14) and (5.15). This leads to

$$
\|\Pi \hat{p}-\tilde{\Pi} \hat{p}\|_{T, P}^{2}=\frac{|\Omega|}{2}\left[\left(c^{T}\right)^{2}+\left(c^{P}\right)^{2}\right] .
$$

Lemma 5.9. Let $c^{T}$ be defined by (5.14); then

$$
\left|c^{T}\right| \leq|\Omega|^{-1 / 2}\left(\sum_{i}\left\|\hat{p}-(\Pi \hat{p})_{i}^{T}\right\|_{L^{2}\left(T_{i}\right)}^{2}\right)^{1 / 2} .
$$

Proof. From (5.14), we have that

$$
|\Omega| c^{T}=\sum_{i}\left|T_{i}\right|(\Pi \hat{p})_{i}^{T}=\sum_{i} \int_{T_{i}}\left[(\Pi \hat{p})_{i}^{T}-\hat{p}\right](\mathbf{x}) d \mathbf{x}
$$

because $\sum_{i} \int_{T_{i}} \hat{p}(\mathbf{x}) d \mathbf{x}=\int_{\Omega} \hat{p}(\mathbf{x}) d \mathbf{x}=0$. Then, a continuous and a discrete Cauchy-Schwarz inequality lead to (5.21) through

$$
|\Omega|\left|c^{T}\right| \leq \sum_{i}\left|T_{i}\right|^{1 / 2}|| \hat{p}-(\Pi \hat{p})_{i}^{T} \|_{L^{2}\left(T_{i}\right)} \leq|\Omega|^{1 / 2}\left(\sum_{i}\left\|\hat{p}-(\Pi \hat{p})_{i}^{T}\right\|_{L^{2}\left(T_{i}\right)}^{2}\right)^{1 / 2} .
$$

So, what remains to evaluate is the $L^{2}$ norm of $(\Pi \hat{p})_{i}^{T}-\hat{p}$ on $T_{i}$.

Lemma 5.10. There exists a constant $C\left(\theta^{*}\right)$ that does not depend on $h$ such that

$$
\left\|\hat{p}-(\Pi \hat{p})_{i}^{T}\right\|_{L^{2}\left(T_{i}\right)} \leq C\left(\theta^{*}\right) h\|\hat{p}\|_{2, T_{i}} .
$$


Proof. First, we split the integral on the subtriangles $t_{i, j, k}$ with vertices $G_{i} M_{j} S_{k}$ (see Fig. 4), where $M_{j}$ is the midpoint of the edge $A_{j} \subset \partial T_{i}$ and $S_{k}$ one of its vertices:

$$
\int_{T_{i}}\left[(\Pi \hat{p})_{i}^{T}-\hat{p}\right]^{2}(\mathbf{x}) d \mathbf{x}=\sum_{t_{i, j, k} \subset T_{i}} \int_{t_{i, j, k}}\left[(\Pi \hat{p})_{i}^{T}-\hat{p}\right]^{2}(\mathbf{x}) d \mathbf{x} .
$$

Let $\pi_{i, j, k} \hat{p}$ be the standard $P^{1}$ Lagrange interpolation of $\hat{p}$ on $t_{i, j, k}$. We have

$$
\left\|\hat{p}-(\Pi \hat{p})_{i}^{T}\right\|_{L^{2}\left(t_{i, j, k}\right)}^{2} \leq 2\left\|(\Pi \hat{p})_{i}^{T}-\pi_{i, j, k} \hat{p}\right\|_{L^{2}\left(t_{i, j, k}\right)}^{2}+2\left\|\pi_{i, j, k} \hat{p}-\hat{p}\right\|_{L^{2}\left(t_{i, j, k}\right)}^{2} .
$$

It is a standard result (see Theorem 3.1.5 of [40]) that there exists a constant $C$, not depending on $t_{i, j, k}$ such that

$$
\left\|\pi_{i, j, k} \hat{p}-\hat{p}\right\|_{L^{2}\left(t_{i, j, k}\right)} \leq \operatorname{Cdiam}\left(t_{i, j, k}\right)^{2}\|\hat{p}\|_{H^{2}\left(t_{i, j, k}\right)},
$$

which evaluates the second term in the right-hand side of (5.24). As far as the first term in the right-hand side of (5.24) is concerned, since $(\Pi \hat{p})_{i}^{T}-\pi_{i, j, k} \hat{p}\left(G_{i}\right)=0$, and since $\pi_{i, j, k} \hat{p}$ is a $P^{1}$ function, we have that, for all $\mathbf{x} \in t_{i, j, k}$

$$
(\Pi \hat{p})_{i}^{T}-\pi_{i, j, k} \hat{p}(\mathbf{x})=\nabla \pi_{i, j, k} \hat{p} \cdot\left(G_{i}-\mathbf{x}\right),
$$

which ensures that $\left|(\Pi \hat{p})_{i}^{T}-\pi_{i, j, k} \hat{p}(\mathbf{x})\right| \leq \operatorname{diam}\left(t_{i, j, k}\right)|| \boldsymbol{\nabla} \pi_{i, j, k} \hat{p} \|$ for all $\mathbf{x} \in t_{i, j, k}$. Thus

$$
\left\|\pi_{i, j, k} \hat{p}-(\Pi \hat{p})_{i}^{T}\right\|_{L^{2}\left(t_{i, j, k}\right)} \leq \operatorname{diam}\left(t_{i, j, k}\right)\left\|\nabla \pi_{i, j, k} \hat{p}\right\|_{L^{2}\left(t_{i, j, k}\right)} .
$$

Using the triangular inequality

$$
\left\|\boldsymbol{\nabla} \pi_{i, j, k} \hat{p}\right\|_{L^{2}\left(t_{i, j, k}\right)} \leq\left\|\boldsymbol{\nabla}\left(\pi_{i, j, k} \hat{p}-\hat{p}\right)\right\|_{L^{2}\left(t_{i, j, k}\right)}+\|\boldsymbol{\nabla} \hat{p}\|_{L^{2}\left(t_{i, j, k}\right)}
$$

and using again Theorem 3.1.5 of [40], we have that there exists a constant $C$ depending only the regularity parameter of the subtriangulation (and thus on $\theta^{*}$ ) such that

$$
\left\|\nabla \pi_{i, j, k} \hat{p}\right\|_{L^{2}\left(t_{i, j, k}\right)} \leq\left(1+\operatorname{Cdiam}\left(t_{i, j, k}\right)\right)\|\hat{p}\|_{H^{2}\left(t_{i, j, k}\right)} .
$$

Gathering (5.23), (5.24), (5.25), (5.26) and (5.27) implies (5.22).

We are now able to estimate the second term in the right-hand side of (5.17)

Proposition 5.11. There exists a constant $C\left(\theta^{*}\right)$ that does not depend on $h$ such that

$$
\|\Pi \hat{p}-\tilde{\Pi} \hat{p}\|_{T, P} \leq C\left(\theta^{*}\right) h\|\hat{p}\|_{2, \Omega} .
$$

Proof. Bounds (5.21) and (5.22) imply that

$$
\left|c^{T}\right| \leq C\left(\theta^{*}\right) h|| \hat{p} \|_{2, \Omega} .
$$

Using (5.20), (5.29) and a similar bound that can be obtained in the same way for $c^{P}$, we obtain (5.28).

Finally, using (5.17), (5.18) and (5.28), we obtain the following theorem:

Theorem 5.12. There exists a constant $C\left(\theta^{*}\right)$ that does not depend on $h$ such that

$$
\|p-\Pi \hat{p}\| \|_{T, P} \leq C\left(\theta^{*}\right) h\left(\|\hat{p}\|_{2, \Omega}+\|\hat{\omega}\|_{2, \Omega}\right) .
$$




\subsection{Error estimate in the $L^{2}$-norm for the velocity}

Once the numerical approximation $\omega=\left(\omega_{i}^{T}, \omega_{k}^{P}\right)_{i \in\left[1, I+J^{\Gamma}\right], k \in[1, K]}$ of the vorticity $\hat{\omega}$ is known, we have to solve (4.15) in order to find the discrete velocity $\mathbf{u}=\left(\mathbf{u}_{j}\right)_{j \in[1, J]}$, with which we define a piecewise constant function $\mathbf{u}_{h}$ by

$$
\mathbf{u}_{h}(\mathbf{x})=\mathbf{u}_{j}, \forall \mathbf{x} \in D_{j}, \forall j \in[1, J] .
$$

In order to estimate the error between $\mathbf{u}_{h}$ and the exact velocity $\hat{\mathbf{u}}$, we first introduce an intermediate discrete velocity $\tilde{\mathbf{u}}=\left(\tilde{\mathbf{u}}_{j}\right)_{j \in[1, J]}$, solution of the discrete div-curl system

$$
\left\{\begin{aligned}
\left(\nabla_{h}^{T, P} \cdot \tilde{\mathbf{u}}\right)_{i, k} & =g_{i, k}^{T, P}, \quad \forall i \in[1, I], \forall k \in[1, K], \\
\left(\nabla_{h}^{T, P} \times \tilde{\mathbf{u}}\right)_{i, k} & =\langle\hat{\omega}\rangle_{i, k}^{T, P}, \quad \forall i \in[1, I], \forall k \notin \Gamma, \\
\tilde{\mathbf{u}}_{j} \cdot \mathbf{n}_{j} & =\sigma_{j}, \quad \forall j \in \Gamma, \\
(\tilde{\mathbf{u}} \cdot \boldsymbol{\tau}, 1)_{\Gamma_{q}, h} & =k_{q}, \quad \forall q \in[1, Q], \\
\sum_{k \in \Gamma_{q}}\left|P_{k}\right|\left(\nabla_{h}^{P} \times \tilde{\mathbf{u}}\right)_{k} & =\sum_{k \in \Gamma_{q}}\left|P_{k}\right|\langle\hat{\omega}\rangle_{k}^{P}, \quad \forall q \in[1, Q],
\end{aligned}\right.
$$

where we have set

$$
\langle\hat{\omega}\rangle_{i}^{T}:=\frac{1}{\left|T_{i}\right|} \int_{T_{i}} \hat{\omega}(\mathbf{x}) d \mathbf{x} \quad \forall i \in[1, I] \quad \text { and } \quad\langle\hat{\omega}\rangle_{k}^{P}:=\frac{1}{\left|P_{k}\right|} \int_{P_{k}} \hat{\omega}(\mathbf{x}) d \mathbf{x} \quad \forall k \in[1, K] .
$$

Note that $\tilde{\mathbf{u}}$ is of course never actually computed (because the exact vorticity $\hat{\omega}$ is not known), but only serves for theoretical reasons. The following triangle inequality holds

$$
\left\|\mathbf{u}_{h}-\hat{\mathbf{u}}\right\|_{0, \Omega} \leq\|\mathbf{u}-\tilde{\mathbf{u}}\|_{D}+\left\|\tilde{\mathbf{u}}_{h}-\hat{\mathbf{u}}\right\|_{0, \Omega} .
$$

A bound of the second term in the right-hand side of (5.32) is given by [25, Th. 5.22]:

Proposition 5.13. If all diamond-cells are convex and under Hypotheses 2.1 and 5.4, there exists a constant $C\left(\theta^{*}\right)$ independent of $h$ such that

$$
\left\|\tilde{\mathbf{u}}_{h}-\hat{\mathbf{u}}\right\|_{0, \Omega} \leq C\left(\theta^{*}\right) h\left(\|g\|_{0, \Omega}+\|\hat{\omega}\|_{0, \Omega}+\|\hat{\phi}\|_{2, \Omega}+\|\hat{\psi}\|_{2, \Omega}\right) .
$$

The next step is the evaluation of the difference $\mathbf{d}_{u}:=\mathbf{u}-\tilde{\mathbf{u}}$, which, using (4.15) and (5.31), and setting $d_{\omega}:=\omega-\langle\hat{\omega}\rangle$, is the solution of

$$
\left\{\begin{aligned}
\left(\nabla_{h}^{T, P} \cdot \mathbf{d}_{u}\right)_{i, k} & =0, \quad \forall i \in[1, I], \forall k \in[1, K], \\
\left(\nabla_{h}^{T, P} \times \mathbf{d}_{u}\right)_{i, k} & =\left(d_{\omega}\right)_{i, k}^{T, P}, \quad \forall i \in[1, I], \forall k \notin \Gamma, \\
\left(\mathbf{d}_{u}\right)_{j} \cdot \mathbf{n}_{j} & =0, \quad \forall j \in \Gamma, \\
\left(\mathbf{d}_{u} \cdot \boldsymbol{\tau}, 1\right)_{\Gamma_{q}, h} & =0, \quad \forall q \in[1, Q], \\
\sum_{k \in \Gamma_{q}}\left|P_{k}\right|\left(\nabla_{h}^{P} \times \mathbf{d}_{u}\right)_{k} & =\sum_{k \in \Gamma_{q}}\left|P_{k}\right|\left(d_{\omega}\right)_{k}^{P}, \quad \forall q \in[1, Q],
\end{aligned}\right.
$$

Proposition 5.14. There exists a constant $C\left(\theta^{*}\right)$ independent of $h$ such that

$$
\|\mathbf{u}-\tilde{\mathbf{u}}\|_{D} \leq C\left(\theta^{*}\right)\|\omega-\langle\hat{\omega}\rangle\|_{T, P}
$$


Proof. We use the discrete Hodge decomposition (see Proposition 3.8) of $\mathbf{d}_{u}$ into $\nabla_{h}^{D} \phi_{\mathbf{d}}+$ $\nabla_{h}^{D} \times \psi_{\mathbf{d}}$. Using Proposition 4.3, we get that $\phi_{\mathbf{d}}$ vanishes. Next, performing like in the proof of Theorem 4.1 in [35], we obtain $\left\|\mathbf{d}_{u}\right\|_{D}^{2}=\left(\nabla_{h}^{T, P} \times \mathbf{d}_{u}, \psi_{\mathbf{d}}\right)_{T, P}$. By definition of the scalar product (3.6), and using the second line in (5.34), it holds that

$$
\begin{gathered}
\left(\nabla_{h}^{T, P} \times \mathbf{d}_{u}, \psi_{\mathbf{d}}\right)_{T, P}=\frac{1}{2} \sum_{i \in[1, I]}\left|T_{i}\right|\left(d_{\omega}\right)_{i}^{T}\left(\psi_{\mathbf{d}}\right)_{i}^{T}+\frac{1}{2} \sum_{k \notin \Gamma}\left|P_{k}\right|\left(d_{\omega}\right)_{k}^{P}\left(\psi_{\mathbf{d}}\right)_{k}^{P} \\
+\frac{1}{2} \sum_{k \in \Gamma_{0}}\left|P_{k}\right|\left(\nabla_{h}^{T, P} \times \mathbf{d}_{u}\right)_{k}^{P}\left(\psi_{\mathbf{d}}\right)_{k}^{P}+\sum_{q \in[1, Q]} \frac{1}{2} \sum_{k \in \Gamma_{q}}\left|P_{k}\right|\left(\nabla_{h}^{T, P} \times \mathbf{d}_{u}\right)_{k}^{P}\left(\psi_{\mathbf{d}}\right)_{k}^{P} .
\end{gathered}
$$

Using boundary condition (3.16) for $\psi_{\mathbf{d}}$, we obtain

$$
\sum_{k \in \Gamma_{0}}\left|P_{k}\right|\left(\nabla_{h}^{T, P} \times \mathbf{d}_{u}\right)_{k}^{P}\left(\psi_{\mathbf{d}}\right)_{k}^{P}=0=\sum_{k \in \Gamma_{0}}\left|P_{k}\right|\left(d_{\omega}\right)_{k}^{P}\left(\psi_{\mathbf{d}}\right)_{k}^{P} .
$$

Moreover, using boundary condition (3.17) and the last line in (5.34), we obtain

$$
\sum_{k \in \Gamma_{q}}\left|P_{k}\right|\left(\nabla_{h}^{T, P} \times \mathbf{d}_{u}\right)_{k}^{P}\left(\psi_{\mathbf{d}}\right)_{k}^{P}=\sum_{k \in \Gamma_{q}}\left|P_{k}\right|\left(d_{\omega}\right)_{k}^{P}\left(\psi_{\mathbf{d}}\right)_{k}^{P}
$$

Thus, gathering (5.36), (5.37), (5.38), and recalling that $\left\|\nabla_{h}^{D} \times \psi_{\mathbf{d}}\right\|_{D}^{2}=\left\|\mathbf{d}_{u}\right\|_{D}^{2}=\left(\nabla_{h}^{T, P} \times\right.$ $\left.\mathbf{d}_{u}, \psi_{\mathbf{d}}\right)_{T, P}$, we get

$$
\left\|\nabla_{h}^{D} \times \psi_{\mathbf{d}}\right\|^{2}=\left(\nabla_{h}^{T, P} \times \mathbf{d}_{u}, \psi_{\mathbf{d}}\right)_{T, P}=\left(d_{\omega}, \psi_{\mathbf{d}}\right)_{T, P} .
$$

Using (5.39), the Cauchy-Schwarz inequality $\left(d_{\omega}, \psi_{\mathbf{d}}\right)_{T, P} \leq\left\|d_{\omega}\right\|_{T, P}\left\|\psi_{\mathbf{d}}\right\|_{T, P}$ and the discrete Poincaré inequality (3.22) for $\psi_{\mathbf{d}}$, allow us to conclude.

Next, it remains to estimate $d_{\omega}$. We first link $\|\omega-\langle\hat{\omega}\rangle\|_{T, P}$ to a previous result by the triangle inequality

$$
\|\omega-\langle\hat{\omega}\rangle\|_{T, P} \leq\|\omega-\Pi \hat{\omega}\|_{T, P}+\|\langle\hat{\omega}\rangle-\Pi \hat{\omega}\|_{T, P} .
$$

The first term in the right-hand side of (5.40) is bounded by (5.13). Bounding the second term requires some more analysis.

Proposition 5.15. Under hypotheses 2.1 and 5.2, there exists a constant $C\left(\theta^{*}\right)$ independent of $h$ such that

$$
\|\langle\hat{\omega}\rangle-\Pi \hat{\omega}\|_{T, P} \leq C\left(\theta^{*}\right) h\|\hat{\omega}\|_{2, \Omega}
$$

Proof. First, we have

$$
\|\langle\hat{\omega}\rangle-\Pi \hat{\omega}\|_{T, P}^{2}=\frac{1}{2}\left[\sum_{i}\left|T_{i}\right|\left(\langle\hat{\omega}\rangle_{i}^{T}-(\Pi \hat{\omega})_{i}^{T}\right)^{2}+\sum_{k}\left|P_{k}\right|\left(\langle\hat{\omega}\rangle_{k}^{P}-(\Pi \hat{\omega})_{k}^{P}\right)^{2}\right] .
$$

Let us consider the first sum in the right-hand side of (5.42); the second sum will be treated in the same way, with however an important modification that will be underlined within the developments that follow. 
Since $\langle\hat{\omega}\rangle_{i}^{T}-(\Pi \hat{\omega})_{i}^{T}$ is a constant on $T_{i}$, we have

$$
\begin{aligned}
\left|T_{i}\right|\left(\langle\hat{\omega}\rangle_{i}^{T}-(\Pi \hat{\omega})_{i}^{T}\right)^{2} & =\int_{T_{i}}\left(\langle\hat{\omega}\rangle_{i}^{T}-(\Pi \hat{\omega})_{i}^{T}\right)^{2} d \mathbf{x} \\
& \leq 2\left(\left\|\hat{\omega}-\langle\hat{\omega}\rangle_{i}^{T}\right\|_{L^{2}\left(T_{i}\right)}^{2}+\left\|\hat{\omega}-(\Pi \hat{\omega})_{i}^{T}\right\|_{L^{2}\left(T_{i}\right)}^{2}\right) .
\end{aligned}
$$

Using arguments similar to those that led to (5.22), we have that

$$
\left\|\hat{\omega}-(\Pi \hat{\omega})_{i}^{T}\right\|_{L^{2}\left(T_{i}\right)} \leq C h\|\hat{\omega}\|_{2, T_{i}} .
$$

Since $T_{i}$ is an open bounded set which is star-shaped with respect to $G_{i}$, there exists a constant $C\left(T_{i}\right)$ only depending on the shape of $T_{i}$, but not on its diameter such that

$$
\left\|\hat{\omega}-\langle\hat{\omega}\rangle_{i}^{T}\right\|_{L^{2}\left(T_{i}\right)} \leq C\left(T_{i}\right) \operatorname{diam}\left(T_{i}\right)\|\nabla \hat{\omega}\|_{L^{2}\left(T_{i}\right)} .
$$

Since $T_{i}$ is convex, a universal constant $C\left(T_{i}\right)$ is given by $\frac{1}{\pi}$, see [41]. Since any dual cell $P_{k}$ is star-shaped with respect to $S_{k}$, a similar inequality holds:

$$
\left\|\hat{\omega}-\langle\hat{\omega}\rangle_{k}^{P}\right\|_{L^{2}\left(P_{k}\right)} \leq C\left(P_{k}\right) \operatorname{diam}\left(P_{k}\right)\|\nabla \hat{\omega}\|_{L^{2}\left(P_{k}\right)} .
$$

However, since $P_{k}$ is not necessarily convex, the estimation of $C\left(P_{k}\right)$ is less obvious, but we may use explicitly computable formulas given, for example, by [42, 43]. These formulas show that $C\left(P_{k}\right)$ only depend on the angles of the subtriangulation mentioned in Hyp. 2.1. Gathering (5.43), (5.44) and (5.45), and similar inequalities on the dual cells $P_{k}$, the upper bound (5.41) is obtained from (5.42).

Now, we are able to estimate the first term in the right-hand side of (5.32). With (5.40), (5.13) and (5.41), we obtain from (5.35) the following proposition:

Proposition 5.16. Under hypotheses 2.1 and 5.2, there exists a constant $C\left(\theta^{*}\right)$ independent of $h$ such that

$$
\|\mathbf{u}-\tilde{\mathbf{u}}\|_{D} \leq C\left(\theta^{*}\right) h\left(\|\hat{\omega}\|_{2, \Omega}+\|\hat{p}\|_{2, \Omega}\right) .
$$

Finally, plugging (5.47) and (5.33) into (5.32) leads to the following theorem

Theorem 5.17. If all diamond cells are convex and under hypotheses 2.1, 5.2 and 5.4, there exists a constant $C\left(\theta^{*}\right)$ independent of $h$ such that

$$
\left\|\mathbf{u}_{h}-\hat{\mathbf{u}}\right\|_{0, \Omega} \leq C\left(\theta^{*}\right) h\left(\|\hat{\omega}\|_{2, \Omega}+\|\hat{p}\|_{2, \Omega}+\|g\|_{0, \Omega}+\|\hat{\phi}\|_{2, \Omega}+\|\hat{\psi}\|_{2, \Omega}\right) .
$$

Remark 5.18. The convergence results obtained in this section are valid on any mesh; however, on some type of meshes, it is possible to prove some superconverge results. They will not be proved here, but it may be shown that if $\hat{\omega}$ and $\hat{p}$ given by Proposition 5.1 belong to $H^{3}(\Omega)$, then, on families of meshes such that there exists a finite number $L$ of sub-domains $\left(\Omega_{\ell}\right)_{\ell \in[1, L]}$ included in $\Omega$ and independent of the mesh step $h$, such that the diamond-cells which are not parallelograms are included in strips $\delta_{\ell}$, having width $C h$ and located along the boundaries of the various $\Omega_{\ell}$, where $C$ is a constant independent of $h$, then $\left\|\nabla_{h}^{D}(p-\Pi \hat{p})\right\|_{D}$ and $\left\|\nabla_{h}^{D}(\omega-\Pi \hat{\omega})\right\|_{D}$ both converge 
to 0 with the order $h^{3 / 2}$, and, using the discrete Poincaré inequality, so do $\left.\| p-\Pi \hat{p}\right) \|_{T, P}$ and $\| \omega-\Pi \hat{\omega}) \|_{T, P}$.

Families of uniformly refined meshes of rectangles, or any (possibly non-conforming) union of such meshes verify such an hypothesis; see for example on Fig. 7 the second family of meshes used in section 6. In that case, each mesh of rectangles is an $\Omega_{\ell}$. Another example of such families of meshes is what is called "homothetically refined triangular meshes", obtained from a coarse triangular mesh by iteratively refining each triangle into four homothetic sub-triangles by joining the midpoints of its edges. In that case, each triangle of the coarse mesh is an $\Omega_{\ell}$.

\section{Numerical results}

In this section, we first test the finite volume method applied to the vorticity-velocity-pressure formulation against known analytical solutions and plot convergence curves (in log-log scale) for several quantities; then we consider the Bercovier-Engelman [44] as well as the Ruas [45] test case.

\subsection{Convergence curves}

First, the data are chosen so that the exact solution is

$$
\hat{\mathbf{u}}=(\exp (x) \cos (\pi y), x \sin (\pi y)+\cos (\pi x))^{T} \text { and } \hat{p}=x y \exp (x) \cos (\pi y) .
$$

The boundary conditions are those given by (4.1).

Three families of increasingly fine meshes are used. The first is a family of standard triangular meshes, used on the non-simply connected domain $\Omega=]-1 / 2 ; 1 / 2\left[{ }^{2} \backslash\right]-1 / 6 ; 1 / 6\left[{ }^{2}\right.$, see Fig. 6 . The second and third families are nonconforming meshes used on the domain $\Omega=]-1 / 2 ; 1 / 2\left[^{2}\right.$. The second family has very localized non-conformities, see Fig. 7, and is obtained in the following way: the first mesh is obtained by dividing the domain into $8 \times 8$ identical squares, and the 4 squares at the center of the mesh are further refined into $4 \times 4$ sub-squares. Then, the subsequent meshes are obtained by dividing each cell of the previous mesh into $2 \times 2$ square cells. The third family has non-conformities spread over the entire domain, see Fig. 8 , since every other cell is refined into $4 \times 4$ sub-cells. Of course, the third family of meshes is not of practical use but illustrates well the ability of the scheme to deal with heavily non-conforming meshes.

We have proved (see Sections 5.1 and 5.2) that $p, \omega$, as well as their gradients converge to the exact solution of the Stokes problem. We are thus interested here in the numerical convergence of $p, \nabla_{h}^{D} p, \omega$ and $\nabla_{h}^{D} \omega$, which we measure by the following errors

$$
(e 0 p)(h):=\frac{\|p-\Pi \hat{p}\|_{T, P}}{\|\Pi \hat{p}\|_{T, P}} \text { and }(e 1 p)^{2}(h):=\frac{\sum_{j}\left|D_{j}\right|\left|\left(\nabla_{h}^{D} p\right)_{j}-(\Pi \nabla \hat{p})_{j}\right|^{2}}{\sum_{j}\left|D_{j}\right|\left|(\Pi \nabla \hat{p})_{j}\right|^{2}},
$$

where $\forall j \in[1, J],(\Pi \nabla \hat{p})_{j}=(\boldsymbol{\nabla} \hat{p})\left(B_{j}\right)$, where $B_{j}$ is the center of gravity of the diamond cell $D_{j}$. The same definitions hold for $\omega$ by replacing $p$ by $\omega$ in the previous formulae. From the numerical results given in [15], we may expect second-order accuracy for $p$ and $\omega$ (although we were able to prove only first-order accuracy in section 5), and first-order accuracy for $\nabla_{h}^{D} p$ and $\nabla_{h}^{D} \omega$ on general meshes. However, on meshes with diamond-cells which are almost all 
parallelograms, which is the case for the second family of meshes, we expect a convergence order of 1.5 for $\nabla_{h}^{D} p$ and $\nabla_{h}^{D} \omega$.

We also proved the convergence of the velocity field $\mathbf{u}$ to the exact solution. The discrete relative $\mathrm{L}^{2}$ error on the diamond cells for the velocity is measured by:

$$
e^{2}(h):=\frac{\sum_{j}\left|D_{j}\right|\left|\mathbf{u}_{j}-(\Pi \hat{\mathbf{u}})_{j}\right|^{2}}{\sum_{j}\left|D_{j}\right|\left|(\Pi \hat{\mathbf{u}})_{j}\right|^{2}},
$$

where $(\Pi \hat{\mathbf{u}})_{j}$ is the value of the exact solution $\hat{\mathbf{u}}$ at the midpoint $M_{j}$ of the edge $A_{j}$ :

$$
\forall j \in[1, J],(\Pi \hat{\mathbf{u}})_{j}=\hat{\mathbf{u}}\left(M_{j}\right) .
$$

We expect first-order convergence of the velocity field on general meshes like those of the first and third families. On the second family of meshes, since almost all diamond cells are parallelograms, we may expect from the numerical results of [25] an order of convergence of at least 1.5. In Finite Element methods, one is also usually concerned with the convergence of $\boldsymbol{\nabla u}$, since $\mathbf{u}$ belongs to $H^{1}(\Omega)$ and since the term $\int_{\Omega} \boldsymbol{\nabla} \mathbf{u}: \nabla \mathbf{v} d \mathbf{x}$ appears in the bilinear form associated with the variational formulation of the Stokes problem. In our formulation, the natural norm induced by the variational formulation is $\left(\|\nabla \cdot \mathbf{u}\|_{L^{2}(\Omega)}^{2}+\|\nabla \times \mathbf{u}\|_{L^{2}(\Omega)}^{2}\right)^{1 / 2}$. Since $\nabla_{h}^{T, P} \cdot \mathbf{u}$ is always exactly imposed through the first equation of (4.15) or (4.16), we measure the errors on the derivatives of $\mathbf{u}$ through the error on $\omega=\nabla_{h}^{T, P} \times \mathbf{u}$.

\subsubsection{Triangular meshes}

We first consider standard triangular meshes, as shown in Fig. 6. On this type of meshes, $\nabla_{h}^{D} p$ and $\nabla_{h}^{D} \omega$ are all first-order accurate, while $p, \omega$ and $\mathbf{u}$ are second-order accurate, as displayed in Fig. 6. Apart from $\mathbf{u}$ which displays superconverge, these are the expected orders of convergence.
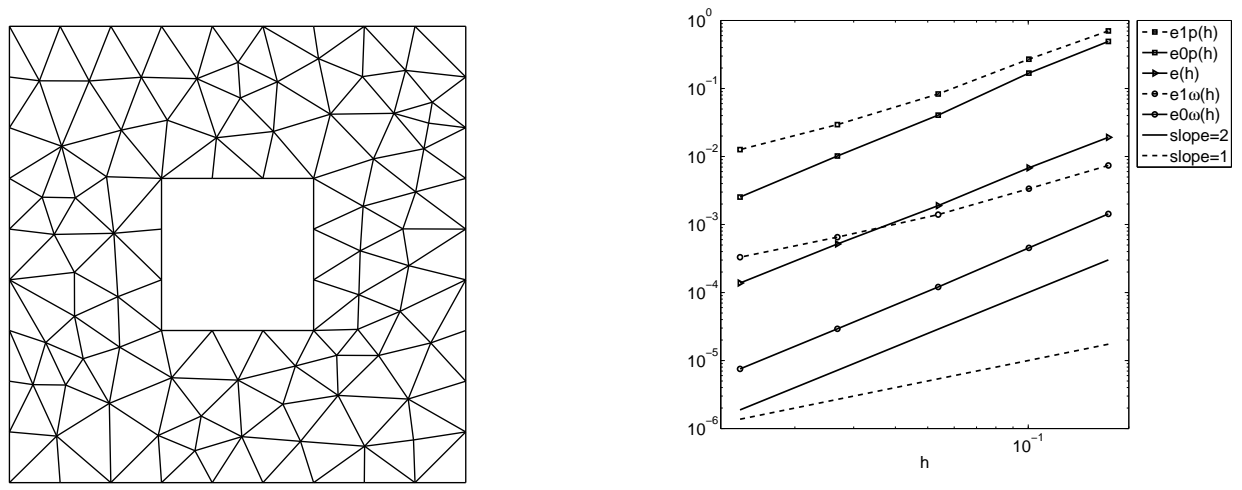

Figure 6: Left: Triangular mesh. Right: Errors. 


\subsubsection{Locally refined meshes}

On the second family of meshes (see Fig. 7), we observe a super-convergence of order 1.5 of $\nabla_{h}^{D} p$ and $\boldsymbol{\nabla}_{h}^{D} \omega$, as expected. Moreover, as far as $\mathbf{u}$ is concerned, we observe in practice an order of convergence which is better than expected since it is slightly lower than 2 . On the third family
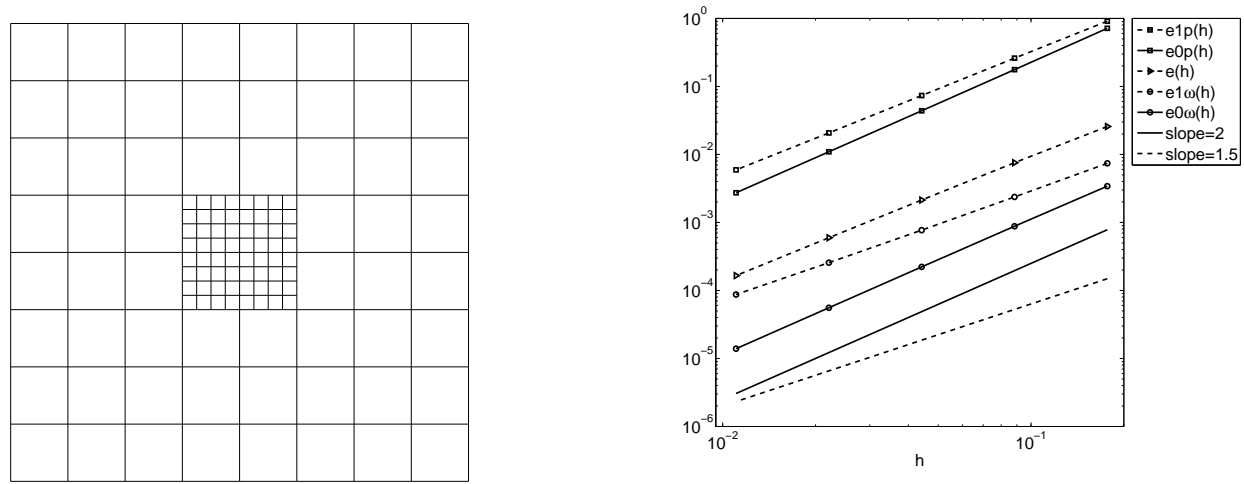

Figure 7: Left: Non-conforming mesh. Right: Errors.

of meshes, see Fig. 8, we recover the same orders of convergence as those obtained on triangular meshes, except for $\mathbf{u}$ which has only first-order convergence.
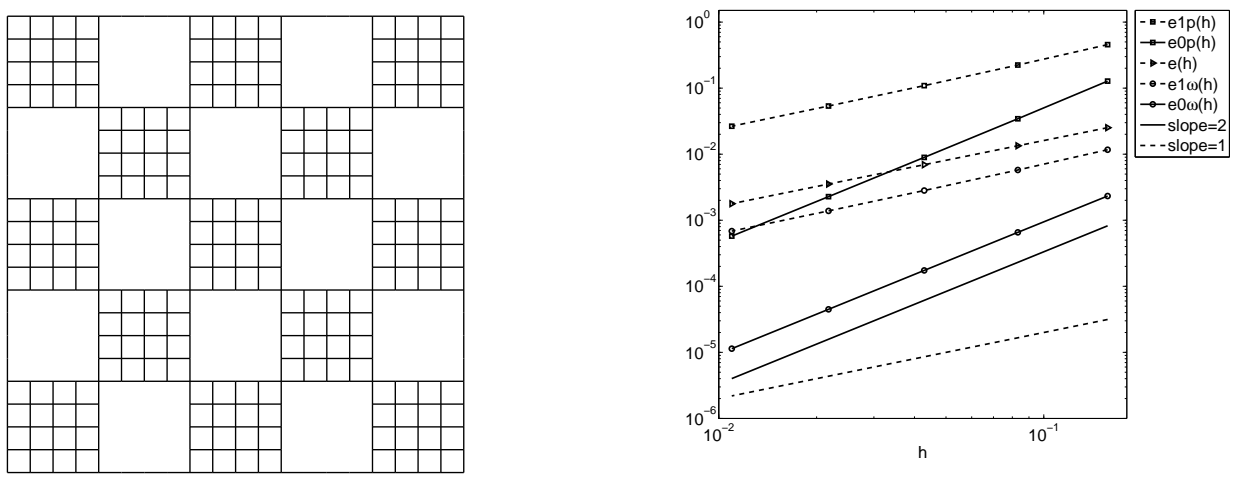

Figure 8: Left: Non-conforming mesh. Right: Errors.

\subsection{The Bercovier-Engelman test case}

The domain of computation is the unit square and the boundary conditions are $\mathbf{u} \cdot \mathbf{n}=0$ on the whole boundary and $\omega=256 y^{2}(y-1)^{2}$ for $x=0$ or $x=1$ and $\omega=256 x^{2}(x-1)^{2}$ for $y=0$ or 
$y=1$, while $\mathbf{f}=\left(f_{1}, f_{2}\right)^{T}$ with

$$
\begin{aligned}
& f_{1}(x, y)=256\left(x^{2}(x-1)^{2}(12 y-6)+y(y-1)(2 y-1)\left(12 x^{2}-12 x+2\right)\right)+(y-1 / 2) \\
& f_{2}(x, y)=-f_{1}(y, x)
\end{aligned}
$$

The exact solution is $\omega=256\left(y^{2}(y-1)^{2}\left(6 x^{2}-6 x+1\right)+x^{2}(x-1)^{2}\left(6 y^{2}-6 y+1\right)\right), p=(x-$ $1 / 2)(y-1 / 2)$ and $\mathbf{u}=\left(u_{1}, u_{2}\right)^{T}$ with

$$
\begin{aligned}
& u_{1}(x, y)=-256 y(y-1)(2 y-1) x^{2}(x-1)^{2} \\
& u_{2}(x, y)=-u_{1}(y, x) .
\end{aligned}
$$

This test case is interesting because in the source term $\mathbf{f}$, the component related to $\boldsymbol{\nabla} \times \boldsymbol{\omega}$ is much larger in magnitude as that related to $\nabla p$, and thus one may wish to know if a numerical scheme is able to calculate the pressure field correctly. This is indeed the case as may be seen from the results displayed on Figure 9 which were computed on a triangular mesh with around 63000 triangles.
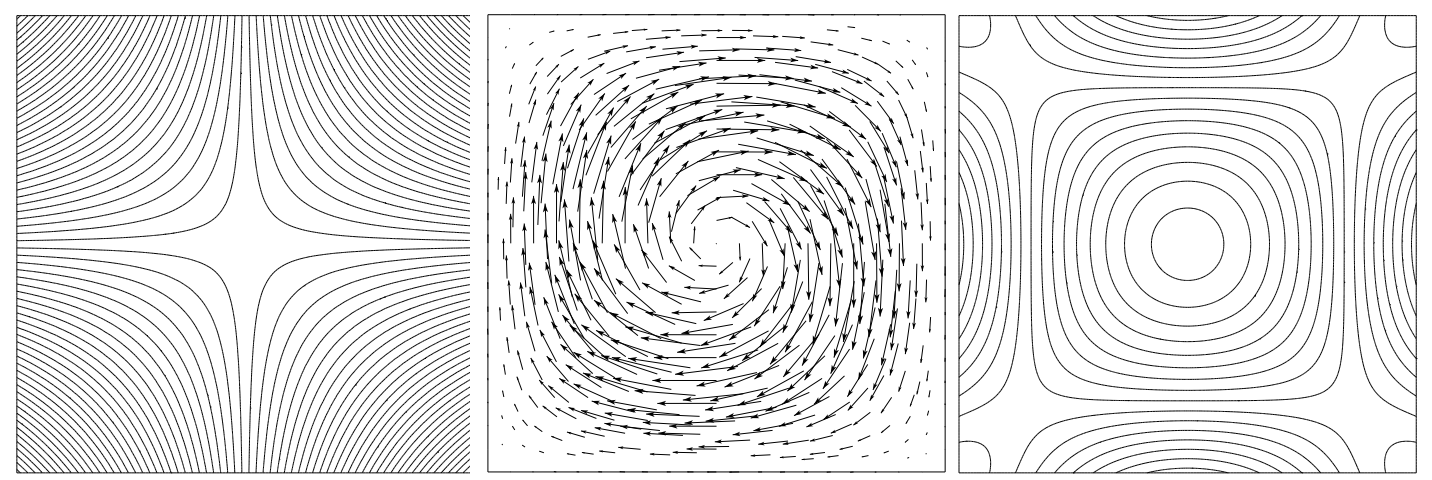

Figure 9: Bercovier-Engelman test. Left: pressure contours (expected extrema : $-1 / 4$ and 1/4, computed extrema: -0.24989 and 0.25003$)$. Center: velocity field. Right: vorticity contours (expected extrema : -16 and 16, computed extrema: -16.001 and 16).

\subsection{The Ruas test-case}

The domain of computation is the unit circle, we use $\mathbf{u} \cdot \mathbf{n}=0$ on the boundary, where we also prescribe the value of the exact vorticity $\hat{\omega}=32-16\left(x^{2}+y^{2}\right)$. Setting $\mathbf{f}=\boldsymbol{\nabla} \times \hat{\omega}$ and $g=0$ yields that $\hat{p}=0$. This test is interesting because a numerical method will compute a discrete pressure which will be, in general, different from 0 , and thus one wishes to know the magnitude of this spurious pressure. However, in the DDFV framework, we wish to emphasize that there is a way of prescribing the right-hand side $\mathbf{f}_{j}$ on each diamond-cell $D_{j}$ in the discrete Helmholtz-Hodge decomposition (Step 1 of our procedure) such that the discrete values $p$ and $\omega$ computed by our scheme are exactly equal to 0 and $\Pi \hat{\omega}$, respectively. Indeed, instead of using 
(4.10), we define $\mathbf{f}_{j}^{D}$ by its scalar products with $\mathbf{n}_{j}$ and $\mathbf{n}_{j}^{\prime}$ in the following way

$$
\mathbf{f}_{j}^{D} \cdot \mathbf{n}_{j}=\frac{1}{\left|A_{j}\right|} \int_{A_{j}} \mathbf{f} \cdot \mathbf{n}_{j} d \sigma \text { and } \mathbf{f}_{j}^{D} \cdot \mathbf{n}_{j}^{\prime}=\frac{1}{\left|A_{j}^{\prime}\right|}\left(\int_{A_{j 1}^{\prime}} \mathbf{f} \cdot \mathbf{n}_{j 1}^{\prime} d \sigma+\int_{A_{j 2}^{\prime}} \mathbf{f} \cdot \mathbf{n}_{j 2}^{\prime} d \sigma\right) .
$$

Then since

$$
\int_{A_{j}} \mathbf{f} \cdot \mathbf{n}_{j}=\int_{A_{j}}(\boldsymbol{\nabla} \times \hat{\omega}) \cdot \mathbf{n}_{j}=\int_{A_{j}}(\boldsymbol{\nabla} \hat{\omega}) \cdot \boldsymbol{\tau}_{j}=\hat{\omega}\left(S_{k 1}\right)-\hat{\omega}\left(S_{k 2}\right)=\left|A_{j}\right|\left(\nabla_{h}^{D} \times \Pi \hat{\omega}\right)_{j} \cdot \mathbf{n}_{j}
$$

and, in the same way

$$
\begin{aligned}
\int_{A_{j 1}^{\prime}} \mathbf{f} \cdot \mathbf{n}_{j 1}^{\prime} d \sigma+\int_{A_{j 2}^{\prime}} \mathbf{f} \cdot \mathbf{n}_{j 2}^{\prime} d \sigma & =\left(\hat{\omega}\left(M_{j}\right)-\hat{\omega}\left(G_{i 1}\right)\right)+\left(\hat{\omega}\left(G_{i 2}\right)-\hat{\omega}\left(M_{j}\right)\right) \\
& =\hat{\omega}\left(G_{i 2}\right)-\hat{\omega}\left(G_{i 1}\right)=\left|A_{j}^{\prime}\right|\left(\nabla_{h}^{D} \times \Pi \hat{\omega}\right)_{j} \cdot \mathbf{n}_{j}^{\prime},
\end{aligned}
$$

we have that $\mathbf{f}_{j}=\left(\nabla_{h}^{D} \times \Pi \hat{\omega}\right)_{j}$ for all $j$; then it follows from the uniqueness of the discrete Helmholtz-Hodge decomposition of the discrete $\mathbf{f}$ that $\omega_{i, k}=(\Pi \hat{\omega})_{i, k}$ and $p_{i, k}^{T, P}=0$. Of course, defining $\mathbf{f}_{j}^{D}$ by (6.2) requires the calculations of the $1 \mathrm{D}$ integrals along the edges; this may be performed by numerical quadrature, up to a user-defined precision. In the Ruas test case, since f is a $P^{1}$ function, the midpoint rule is enough to calculate the integrals exactly. We checked that the computed vorticity is exactly equal to $\Pi \hat{\omega}$ and that the computed pressure vanishes, up to the linear solver precision. The vorticity and the velocity fields are displayed on Figure 10.
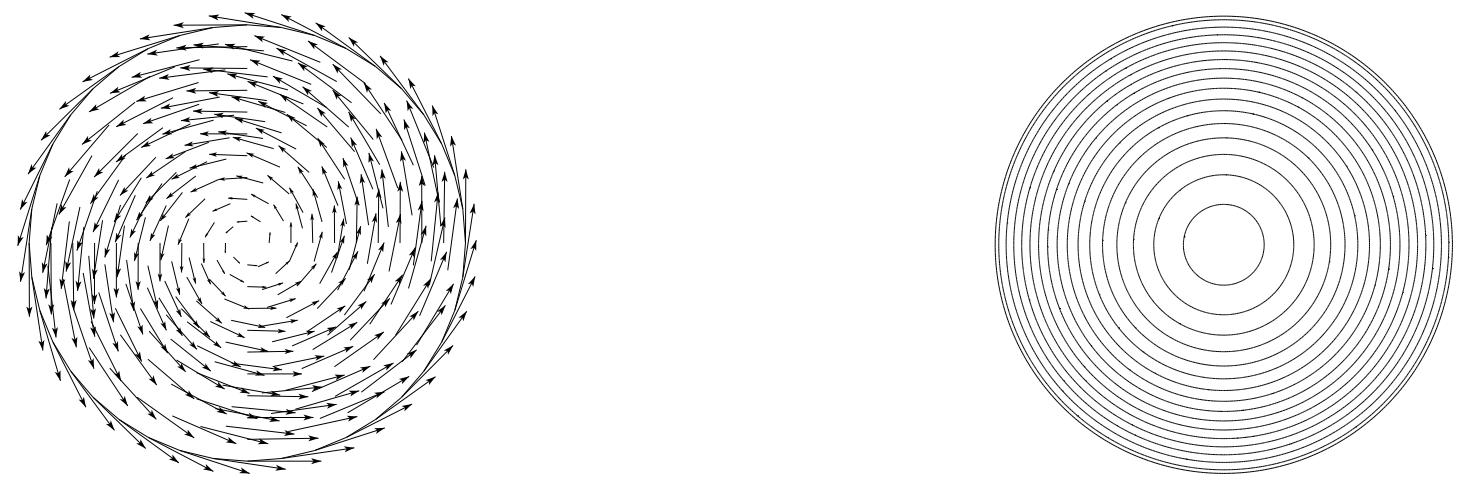

Figure 10: Ruas test. Left: velocity field. Right: vorticity contours.

\section{Conclusion}

We have proposed a discrete duality finite volume method for the two-dimensional Stokes equations with the non-standard boundary conditions (4.1) to (4.4). These non-standard boundary 
conditions are treated through the vorticity-velocity-pressure formulation of the Stokes equations, for which the finite volume method was successfully applied over unstructured and nonconforming meshes. Numerical results show a first-order convergence for the velocity, the pressure gradient and the vorticity gradient and a second order convergence for the pressure and the vorticity, while a superconvergence order of 1.5 for the pressure gradient and the vorticity gradient and an order two for the velocity are obtained on regular (but possibly locally non-conforming) meshes. Some of these convergence orders were proved through the theoretical analysis we led in this paper, while only suboptimal orders were obtained for pressure and vorticity on general meshes and for velocity on regular meshes.

\section{References}

[1] F. Dubois, M. Salaün, and S. Salmon, Vorticity-velocity-pressure and stream functionvorticity formulations for the Stokes problem, J Math Pures Appl 82 (2003), 1395-1451.

[2] C. Bègue, C. Conca, F. Murat, and O. Pironneau, "Les équations de Stokes et de NavierStokes avec des conditions aux limites sur la pression," In Nonlinear Partial Differential Equations and their Applications, Collège de France Seminar Vol. IX, 1988, p. 179-264.

[3] V. Girault, Incompressible Finite Element Methods for Navier-Stokes Equations with Nonstandard Boundary Conditions in $\mathbb{R}^{3}$, Math Comput 183 (1988), 55-74.

[4] M. Amara, D. Capatina-Papaghiuc, E. Chacón-Vera, and D. Trujillo, Vorticity-velocitypressure formulation for Navier-Stokes equations, Comput Vis Sci 6 (2004), 47-52.

[5] M. Amara, E. Chacón-Vera, and D. Trujillo, Vorticity-velocity-pressure formulation for the Stokes equations, Math Comput 73 (2004), 1673-1697.

[6] M. Azaïez, C. Bernardi, and N. Chorfi, Spectral discretization of the vorticity, velocity and pressure formulation of the Navier-Stokes equations, Numer Math 104 (2006), 1-26.

[7] F. Dubois, Vorticity-velocity-pressure formulation for the Stokes problem, Math Meth Appl Sci 25 (2002), 1091-1191.

[8] F. Dubois, M. Salaün, and S. Salmon, First vorticity-velocity-pressure numerical scheme for the Stokes problem, Comput Meth Appl Mech Eng 192 (2003), 4877-4907.

[9] H. Abboud, F. El Chami, and T. Sayah, A priori and a posteriori estimates for threedimensional Stokes equations with nonstandard boundary conditions, Numer Methods Partial Differ Equations 28 (2012), 1178-1193.

[10] C. Bernardi and N. Chorfi, Spectral discretization of the vorticity, velocity and pressure formulation of the Stokes equations, SIAM J Numer Anal 44 (2006), 826-850.

[11] M.M.J. Proot and M.I. Gerrtisma, Least-Squares spectral elements applied to the Stokes problem, J Comput Phys 181 (2002), 454-477.

[12] F.H. Harlow and F.E. Welch, Numerical calculations of time dependent viscous incompressible flow of fluids with a free surface, Phys Fluids 8 (1965), 2182-2189. 
[13] R.A. Nicolaides, T.A. Porshing, and C.A. Hall, "Covolume methods," In computational fluid dynamics review, M. Hafez and K. Oshma (Editors), John Wiley and sons, 1995, p. 279-299.

[14] S. Delcourte, K. Domelevo, and P. Omnes, "Discrete duality finite volume method for second order elliptic problems," In Finite Volumes for Complex Applications IV, F. Benkhaldoun, D. Ouazar and S. Raghay (Editors), Hermes Science publishing, 2005, p. 447-458.

[15] K. Domelevo and P. Omnes, A finite volume method for the Laplace equation on almost arbitrary two-dimensional grids, Math Model Numer Anal 39 (2005), 1203-1249.

[16] F. Hermeline, A finite volume method for the approximation of diffusion operators on distorted meshes, J Comput Phys 160 (2000), 481-499.

[17] F. Hermeline, Approximation of diffusion operators with discontinuous tensor coefficients on distorted meshes, Comput Methods Appl Mech Eng 192 (2003), 1939-1959.

[18] B. Andreianov, F. Boyer, and F. Hubert, Discrete duality finite volume schemes for LerayLions-type elliptic problems on general 2D meshes, Numer Methods Partial Differ Equations 23 (2007), 145-195.

[19] F. Boyer and F. Hubert, Finite volume method for 2D linear and nonlinear elliptic problems with discontinuities, SIAM J Numer Anal 46 (2008), 3032-3070.

[20] Y. Coudière and F. Hubert, A 3D discrete duality finite volume method for nonlinear elliptic equations, SIAM J Sci Comput 33 (2011), 1739-1764.

[21] Y. Coudière and G. Manzini, The discrete duality finite volume method for convectiondiffusion problems, SIAM J Numer Anal 47 (2010), 4163-4192.

[22] B. Andreianov, M. Bendahmane, K. H. Karlsen, and C. Pierre, Convergence of discrete duality finite volume schemes for the cardiac bidomain model, Networks and Heterogeneous Media 6 (2011), 195-240.

[23] Y. Coudière, C. Pierre, O. Rousseau, and R. Turpault, A 2D/3D Discrete Duality Finite Volume Scheme. Application to ECG simulation, Int J Finite Volumes 6 (2009), electronic only.

[24] C. Chainais-Hillairet, Discrete duality finite volume schemes for two-dimensional driftdiffusion and energy-transport models, Internat J Numer Methods Fluids 59 (2009), 239 257.

[25] S. Delcourte, K. Domelevo, and P. Omnes, A discrete duality finite volume approach to Hodge decomposition and div-curl problems on almost arbitrary two-dimensional meshes, SIAM J Numer Anal 45 (2007), 1142-1174.

[26] F. Hermeline, S. Layouni, and P. Omnes, A finite volume method for the approximation of Maxwell's equations in two space dimensions on arbitrary meshes, J Comput Phys 227 (2008), 9365-9388. 
[27] S. Delcourte, Développement de méthodes de volumes finis pour la mécanique des fluides, Ph.D. Thesis (in French), University of Toulouse III, France, 2007. Available at http://tel.archives-ouvertes.fr/tel-00200833/fr/

[28] S. Krell, Stabilized DDFV Schemes for Stokes Problem with Variable Viscosity on General 2D Meshes, Numer Methods Partial Differential Eq (2011), 1666-1706.

[29] S. Krell, Finite volume method for general multifluid flows governed by the interface Stokes problem, Math Models Methods Appl Sci 22 (2012), 1150025, 35 p.

[30] S. Krell and G. Manzini, The discrete duality finite volume method for Stokes equations on three-dimensional polyhedral meshes, SIAM J Numer Anal 50 (2012), 808-837.

[31] P. Blanc, R. Eymard, and R. Herbin, A staggered finite volume scheme on general meshes for the generalized Stokes problem in two space dimensions, Int J Finite Volumes 2 (2005), electronic only.

[32] R. Eymard and R. Herbin, A staggered finite volume scheme on general meshes for the Navier-Stokes equations in two space dimensions, Int J Finite Volumes 2 (2005), electronic only.

[33] R. Eymard, J.C. Latché, and R. Herbin, On a stabilized collocated finite volume scheme for the Stokes problem. Math Model Numer Anal 40 (2006), 501-527.

[34] R. Eymard, J.C. Latché, and R. Herbin, Convergence analysis of a colocated finite volume scheme for the incompressible Navier-Stokes equations on general 2 or 3D meshes, SIAM J Numer Anal 45 (2007), 1-36.

[35] A.H. Le and P. Omnes, Discrete Poincaré inequalities for arbitrary meshes in the discrete duality finite volume context, ETNA, Electronic Transactions on Numerical Analysis 40 (2013), 94-119.

[36] S. Heib, Nouvelles discrétisations non structurées pour des écoulements de fluides à incompressibilité renforcée (in French), Ph.D. Thesis, Université Pierre et Marie Curie, Paris, France, 2002.

[37] C. Bernardi and F. Hecht, More pressure in the finite element discretization of the Stokes Problem, Math Model Numer Anal 34 (2000), 953-980.

[38] M. Crouzeix and P.-A. Raviart, Conforming and nonconforming finite element methods for solving the stationary Stokes equations, RAIRO Anal Numer 7 (1973), 33-76.

[39] V. Girault and P.-A. Raviart, Finite Element Methods for Navier-Stokes Equations, Springer-Verlag, Berlin, 1986.

[40] P.G. Ciarlet, The finite element method for elliptic problems, North Holland publishing company, 1978.

[41] L.E. Payne, and H.F. Weinberger, An optimal Poincaré inequality for convex domain. Arch Rational Mech Anal 5 (1960), 286-292. 
[42] C. Carstensen and S. Funken, Constants in Clément-interpolation error and residual based $a$ posteriori estimates in finite element methods, East-West J Numer Math 8 (2000), 153-175.

[43] R. Verfürth, Error estimates for some quasi-interpolation operators, Math Model Numer Anal 33 (1999), 695-713.

[44] M. Bercovier and M. Engelman, A finite element for the numerical solution of viscous incompressible flows, J Comput Phys 30 (1979), 181-201.

[45] V. Ruas, Méthodes vitesse-tourbillon pour les équations de Navier-Stokes, in: Actes du 29ème congrès d'Analyse Numérique, Canum'97 (1997), 329-330. 